\title{
Use of next generation sequencing to compare simple habitat and species level differences in the gut microbiota of an invasive and native freshwater fish species
}

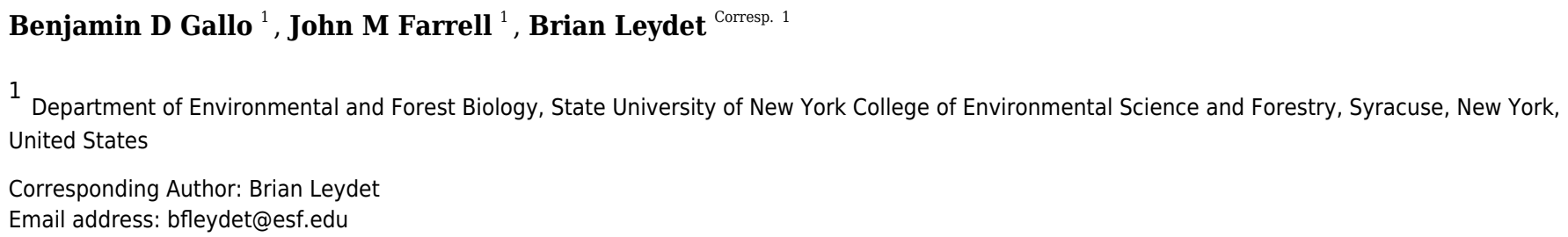

Research on the gut microbiome of host organisms has rapidly advanced with next generation sequencing (NGS) and high-performance computing capabilities. Nonetheless, gut microbiome research has focused on mammalian organisms in laboratory settings, and investigations pertaining to wild fish gut microbiota remain in their infancy. We applied a procedure (available at https://github.com/bngallo1994) for sampling of the fish gut for use in NGS to describe microbial community structure. Our approach allowed for high bacterial OTU diversity coverage ( $>99.7 \%$, Good's Coverage) that led to detection of differences in gut microbiota of an invasive (Round Goby) and native (Yellow Bullhead) collected from the upper St. Lawrence River, an environment where the gut microbiota of fish had not previously been tested. Additionally, results revealed habitat level differences in in gut microbiota using two distance metrics (Unifrac, Bray-Curtis) between nearshore littoral and offshore profundal collections of Round Goby. Species and habitat level differences in intestinal microbiota may be of importance in understanding individual and species variation and its importance in regulating fish health and physiology. 
1 Use of next generation sequencing to compare simple habitat and

2 species level differences in the gut microbiota of an invasive and

3 native freshwater fish species

4

5

6

7 Benjamin D. Gallo, John M. Farrell, Brian F. Leydet

8

$9{ }^{1}$ Department of Environmental \& Forest Biology, State University of New York College of

10 Environmental Science and Forestry, Syracuse, NY, USA

11

12 Corresponding Author:

13 Brian F. Leydet

141 Forestry Drive, Syracuse, NY 13210, USA

15 Email address: bfleydet@esf.edu 


\section{Abstract}

38 Research on the gut microbiome of host organisms has rapidly advanced with next generation 39 sequencing (NGS) and high-performance computing capabilities. Nonetheless, gut microbiome research has focused on mammalian organisms in laboratory settings, and investigations pertaining to wild fish gut microbiota remain in their infancy. We applied a procedure (available at https://github.com/bngallo1994) for sampling of the fish gut for use in NGS to describe microbial community structure. Our approach allowed for high bacterial OTU diversity coverage ( $>99.7 \%$, Good's Coverage) that led to detection of differences in gut microbiota of an invasive (Round Goby) and native (Yellow Bullhead) collected from the upper St. Lawrence River, an environment where the gut microbiota of fish had not previously been tested. Additionally, results revealed habitat level differences in in gut microbiota using two distance metrics (Unifrac, Bray-Curtis) between nearshore littoral and offshore profundal collections of Round Goby. Species and habitat level differences in intestinal microbiota may be of importance in understanding individual and species variation and its importance in regulating fish health and physiology.

\section{Introduction}

Bacterial communities inhabiting the alimentary canal of organisms, often referred to as the host's "gut microbiome", have become a focal area of research over the last decade (Gallo et al. 2020). Studies show that gut microbiota can greatly influence host growth and development (Lozupone et al. 2012), behavior (Johnson and Foster 2018), and immune system function (Colombo et al. 2015). To date, the majority of gut microbiome research has focused on mammals as model organisms for understanding vertebrate microbial communities (Sullam et al. 2012). Mammals though, comprise a relatively small proportion of the total vertebrate diversity, whereas fish represent $\sim 50 \%$ (Sullam et al. 2012). Nonetheless, little is known surrounding the ecology of host-borne microbes in fish, particularly what factors drive patterns of bacterial colonization and community assemblage (Tarnecki et al. 2017). Because an organism's gut microbiome can influence many aspects of host physiology, describing the relative abundance of various microbes is an important first step in delineating organism and/or communities that either benefit or harm the host.

Several factors are known to modulate gut microbiota composition in fishes, including host species/genetics (Li et al. 2012; Li et al. 2014), feeding habits (Michl et al. 2017), trophic 
68 levels (Liu et al. 2016), disease prevalence in the host population (Hennersdorf et al. 2016), and

69 environmental variables including habitat and husbandry practices (Dehler et al. 2017; Wu et al.

70 2012). Recent research on euryhaline fish has indicated that habitat salinity also plays a

71 significant role influencing the dominant gut microbiota (Schmidt et al. 2015). Additionally,

72 laboratory studies investigating the gut microbiota of Zebrafish Danio rerio demonstrate

73 taxonomic similarities despite being raised in different aquaculture facilities (Roeselers et al.

74 2011). However, Zebrafish gut microbiota was also shown to differ temporally during ontogeny, 75 highlighting the dynamic nature of gut-borne microbial communities (Stephens et al. 2016).

76 Additional research is warranted to understand how gut microbial communities develop in nature

77 in order to elucidate the beneficial and deleterious interactions between gut microbes and fish 78 host.

79

80

81

82

83

84

85

86

87

88

89

90

91

\section{Materials \& Methods}

93

94

95

96

97

98

The objective of this study was to adapt a mammalian-based gut microbiome sampling and sequencing protocol to explore the gut microbiota from two fish species from the upper St. Lawrence River. We collected fish mucosal digesta to test for differences in the autochthonous gut microbiome of Round Goby (Neogobius melanostomus) and Yellow Bullhead (Ameiurus natalis). Yellow Bullhead, a native species to the upper St. Lawrence River, consumes small fish and crustaceans (Stegemann 1989) whereas the invasive Round Goby diet is often dominated by invasive Zebra Mussels Dreissena polymorpha (Ray and Corkum 1997). Furthermore, Round Goby generally prefer hard substrate in both shallow and deepwater habitats (Charlebois et al. 2001) while Yellow Bullhead prefer soft substrates in vegetated areas of shallow lakes, reservoirs, and streams (Stegemann 1989). These diet and habitat differences, in addition to known interspecies variation in fish gut microbiota ( $\mathrm{Li}$ et al. 2012) provide a scenario for testing expected differences with the NGS workflow.

Data Collection \& Field Processing. - Collection of fish took place on the upper St. Lawrence River and its tributaries in Clayton, NY (Fig. 1). All specimens were collected through volunteer angling, baited minnow traps, or fine mesh hoopnets and under permit from the New York State Department of Environmental Conservation (license \#354). Capture date, total length (mm), and total weight (g) were recorded for each captured fish (Supplementary Table S1). Euthanasia of animals followed approved protocols outlined by the American Veterinary Association (AVMA)

Peer] reviewing PDF | (2020:02:45747:2:0:NEW 24 Sep 2020) 
99 and the American Fisheries Society (AFS) through an overdose of Tricaine methansulfonate

$100(400+\mathrm{mg} / \mathrm{L})$ or blunt cranial concussion (State University of New York College of

101 Environmental Science and Forestry's Institutional Animal Care and Use Committee protocol

102 \#180202). All fish were euthanized within approximately 3 hours of capture and minnow traps /

103 hoopnets were allowed to soak for a maximum of 18-24 hours. There was no mixing of species

104 or within species by sampling location prior to euthanasia to ensure no sharing of water and

105 potential transfer of microbiota. Fish gut microbiota comparisons involved eight Round Goby

106 captured at Governors Island (shallow littoral: $<2 \mathrm{~m}$ water depth) and seven Round Goby at the

107 Eagle Wings Islands (deepwater profundal: $>15 \mathrm{~m}$ water depth). Seven Yellow Bullhead were

108 also sampled in French Creek (coastal wetland tributary: <1m water depth). Due to sampling

109 habitat limitations for Yellow Bullhead, sampling took place within only one site, and thus

110 Yellow Bullhead were only utilized for inter-species analyses. Round Goby samples from the

111 two separate habitats (Governors Island and Eagle Wings Islands) were combined for inter-

112 species gut microbiota analysis but analyzed separately when testing for inter-habitat gut

113 microbiota associations.

114 After euthanasia, the integument of each fish was surface sterilized by rinsing in a bath of

$11595 \%$ ethanol prior to dissection. All sample collection methods took place at room temperature

$116\left(\sim 20^{\circ} \mathrm{C}\right)$. Fish were dissected with a posterior incision near the pectoral fin origin to the

117 urogenital opening. Approximately $25 \mathrm{mg}$ of hindgut tissue was aseptically removed from each

118 fish using flame-sterilized dissecting scissors and/or scalpels. Digesta was manually cleared from

119 the intestinal tract. Samples were then gently washed with a stream of sterile $0.05 \mathrm{M}$ phosphate-

120 buffered saline (PBS) removing residual digesta while leaving autochthonous bacteria in the

121 hindgut. All samples were stored in $1.75 \mathrm{~mL}$ of nucleic acid preservation buffer (NAP; 0.019 M

122 ethylenediaminetetraacetic acid (EDTA) disodium salt dihydrate, $0.018 \mathrm{M}$ sodium citrate

123 trisodium salt dehydrate, $3.8 \mathrm{M}$ ammonium sulfate, $\mathrm{pH}$ 5.2) allowing for ambient room

124 temperature storage in sterile $2.0 \mathrm{~mL}$ microcentrifuge tubes (Camacho-Sanchez et al. 2013).

125 Samples were stored for 4-8 weeks prior to DNA extraction. Previous examination of NAP

126 buffer showed similar, if not superior, bacterial DNA preservation compared to commonly

127 employed commercial buffers in comparative microbiome analyses (Menke et al. 2017). 
129 Laboratory Processing. - DNA was extracted using the E.Z.N.A ${ }^{\circledR}$ Tissue DNA Kit (Omega 130 Bio-Tek, Norcross, GA) following the manufacturers protocol, except that an overnight (15-17 131 hours) tissue lysis step was employed to assist in complete intestinal tissue digestion. Extracted 132 DNA was stored at $-20^{\circ} \mathrm{C}$ until PCR was performed. To selectively amplify bacterial DNA 133 extracted from the hindgut samples, PCR was conducted using 16S V6-V8 rRNA primers 134 (B969F and BA1406R (Comeau et al. 2011); Integrated DNA Technologies, Coralville, Iowa 135 52241) fused with unique barcodes and Illumina ${ }^{\circledR}$ adapter sequences. Employing this method 136 (primers fused with NGS barcodes and adaptor sequences) resulted in significant cost savings 137 and eliminated further sample processing and amplification steps (see Comeau et al. 2017). The 138 PCR master mix was created following Comeau et al. (2017) with the slight modification of 139 using Q5® High-Fidelity Taq polymerase (New England Biolabs, Ipswich, MA 01938). The 140 PCR cycling protocol was as follows: initial denaturation of $95^{\circ} \mathrm{C}$ for 30 seconds, followed by 35 141 cycles of $95^{\circ} \mathrm{C}$ for 30 seconds, $55^{\circ} \mathrm{C}$ for 30 seconds, $72^{\circ} \mathrm{C}$ for 30 seconds, and a final extension 142 step of $72^{\circ} \mathrm{C}$ for 5 minutes. PCR amplicons ( $\left.\sim 60 \mathrm{bp}\right)$ were verified by gel electrophoresis on a $1432 \%$ agarose gel. Amplicon cleanup and NGS preparation took place using the Agencourt 144 AMPure XP PCR purification kit (Agencourt Biosciences, Beverly, MA) and following 145 manufacturer's protocol. NGS libraries were quantified via the Quant-iT dsDNA HS Assay 146 (Invitrogen, Carlsbad, CA) following the assay's standard protocol. Fluorescence was read on a 147 Biotek ${ }^{\circledR}$ Synergy 2 plate reader (Agilent Technologies, Santa Clara, CA) and samples were 148 subsequently converted from $n{ }^{*} \mu 1^{-1}$ to $\mathrm{nM}$. The NGS library was diluted to a final concentration 149 of $4 \mathrm{nM}$ and the normalized libraries were pooled. For loading on the sequencer, $5 \mu 1$ of the 150 library pool was added to $5 \mu \mathrm{l}$ freshly prepared $0.2 \mathrm{M}$ sodium hydroxide, mixed well, and 151 incubated at ambient temperature for 5 minutes. This was followed by an addition of $990 \mu 1$ of 152 pre-chilled Illumina ${ }^{\circledR}$ HT1 buffer, creating a final $20 \mathrm{pM}$ library concentration. The prepared 153 library was sequenced on an Illumina ${ }^{\circledR}$ MiSeq $^{\mathrm{TM}}$ (San Diego, CA) using 2x300 v3 chemistry and 154 a 10\% PhiX spike at the SUNY Molecular Analysis Core (SUNYMAC) at SUNY Upstate 155 Medical University (http://www.upstate.edu/sunymac/).

156

157 Dataset organization for Analyses. - For all described analyses, Round Goby and Yellow 158 Bullhead samples were organized into two distinct datasets: (1) gut microbiota vs. fish species 159 (15 Round Goby vs. 7 Yellow Bullhead) and (2) gut microbiota vs. fish habitat (7 Eagle Wings 
160 Islands Round Goby vs. 8 Governors Island Round Goby). All quality filtering, OTU clustering, 161 and multivariate comparison procedures were identical between group analyses.

162

163 Sequenced Data Processing and Analysis. - Raw reads have been deposited with links to 164 BioProject accession number PRJNA528762 in the NCBI BioProject database

165 (https://www.ncbi.nlm.nih.gov/bioproject/). Sequenced libraries were demultiplexed in MiSeq ${ }^{\mathrm{TM}}$

166 Reporter v2.6 and FASTQ files were processed using USEARCH v.11.0.667 (Edgar 2013).

167 FASTQ sequences were stitched and filtered to the approximate size of the V6-V8 region of the 168 bacterial 16S rRNA gene (400-600 bp length; Comeau et. al 2017). USEARCH was used to trim 169 primer regions and remove chimeric and low-quality sequences. Next, reads were merged based 170 on similarity (400-600 bp in length, had $\leq 5$ nucleotide differences, and were $\geq 90 \%$ similar).

171 Sequences were filtered with the maximum expected errors per sequence $\leq 0.5$. These cutoffs

172 followed default or more stringent parameters as outlined in the USEARCH guide

173 (https://www.drive5.com/usearch/). Filtered reads were subsequently preclustered by size (99\%

174 similarity, maximum differences $\leq 4$ ) and then clustered into Operational Taxonomic Units

175 (OTUs) based on 97\% similarity using the UPARSE algorithm (Edgar 2013). USEARCH

176 filtering and subsequent OTU clustering was conducted with singleton data (OTUs with single

177 DNA sequence occurrence), as procrustes analysis revealed non-significant differences between

178 singleton and non-singleton multivariate ordinations (package "vegan", function "protest()",

179 Procrustes analysis: $S S=2.47 \times 10^{-4}$ (Species Comparison) \& $1.66 \times 10^{-4}$ (Habitat Comparison), $180 P \leq 0.001$ (Species Comparison) $\& P \leq 0.001$ (Habitat Comparison); Supplemental Fig. S2 \&

181 S3). All USEARCH scripts utilized in these analyses can be retrieved from our GitHub page

182 (https://github.com/bngallo1994)

The USEARCH generated OTU tables were modified into a shared compatible file and uploaded into Mothur v.1.39.5 (Schloss et al. 2009). Rarefaction files were subsequently created using the Mothur MiSeq ${ }^{\mathrm{TM}}$ Standard Operating Procedure (https://www.mothur.org/wiki/MiSeq_SOP). OTU tables were modified to make rarefaction files to estimate species richness. Because of error rates, singleton calls using NGS platforms can be interpreted as potential sequencing artifacts (Brown et al. 2015). Nonetheless, subsequent analysis from the most dominant OTU's in each dataset revealed negligible differences in community structure between singleton-included and excluded microbiota matrices (data not 
191 shown). Rarefaction curves utilizing singleton OTUs thus served as a maximum estimate of total 192 species richness are presented in this manuscript.

193 The SILVA 138 high quality ribosomal RNA database was used to determine the identity 194 of the most abundant OTUs in each fish species (Quast et al. 2013). We queried all OTU16S 195 rRNA sequences using SILVA's Alignment, Classification and Tree Service (ACT) web module 196 (https://www.arb-silva.de/aligner/) (Pruesse et al. 2012). OTU sequences were classified with a 197 minimum query sequence identity of $95 \%$ and 10 neighbors per query sequence

The SILVA generated taxa information was subsequently combined with the USEARCH 199 OTU table and sample metadata into a single phyloseq class object using the bioconductor

200 package 'Phyloseq' (McMurdie and Holmes 2013). Two phyloseq objects were created - one for

201

202

203

204 205

206

207

208

209

210

211

212

213

214

215

216

217

218

219

220

221 the Round Goby vs. Yellow Bullhead Species comparison, and one for the Governor's Island vs. Eagle Wings comparison for Round Goby. Additionally, we analyzed the top 10 OTUs from each dataset at normalized sequencing depth (species comparison: 41,250 sequences/sample; habitat comparison: 40,972 sequences/sample) to determine their average relative abundance in Round Goby and Yellow Bullhead. Using the same strategy, we determined the identity and relative abundance of the top 10 OTUs within each Round Goby habitat group (Eagle Wings Islands vs. Governors Island).

To estimate the microbial community coverage, OTU tables and rarefaction data files were analyzed using the entropart (v 1.5-3; Marcon 2018), ggplot2 (v 3.0.0; Wickham 2016), and vegan (v 2.5.2; Oksanen et al. 2018) packages of the R statistical software (R Core team 2017). Rarefaction analyses were paired with the calculation of Good's Coverage using the Chao method to provide a description of rarefaction in terms of the total sampled OTU diversity (Good 1953; Chao 1984; Larsen 2014). Datasets were rarefied to normalized sequencing depth for comparisons of microbial relative abundances between samples. The instantaneous slope of each rarefaction curve was determined at this normalized depth (Hurlbert 1971). To estimate OTU loss through normalization, we also compared the normalized species richness to the observed richness collected at each sample's maximum sequencing depth (Chao and Jost 2012). These analyses quantitatively assessed our ability to detect a representative coverage of microbial DNA from wild caught Round Goby and Yellow Bullhead.

A number of $\alpha$-diversity metrics were also calculated for all Round Goby and Yellow Bullhead samples. The Observed, Chao1, Shannon, and Simpson indices were calculated to 
222 estimate $\alpha$-diversity between the two fish species and within Round Goby by habitat.

223 Significance between groups was tested from each index with Wilcoxon rank-sum tests (Mann-

224 Whitney) using the Benjamini and Hochberg (1995) p-value correction method.

225 Non-metric multidimensional scaling (NMDS) was employed to visualize dissimilarity in 226 datasets between samples. NMDS (R package "vegan", function "metaMDS") ordination was 227 computed for both normalized habitat and species datasets. NMDS scaling (Kruskal 1964) was 228 used to visualize the Bray-Curtis dissimilarity (Bray and Curtis 1957) between samples using 229 default permutations $(\mathrm{n}=20)$ in the 'metaMDS' function in the vegan. Permutational multivariate 230 analysis of variance using distance matrices (PERMANOVA, package "vegan”, function 231 "adonis"; 999 permutations) was employed to assess significant differences $(\alpha=0.05)$ between 232 the two datasets: (species comparison = Round Goby and Yellow Bullhead; habitat comparison = 233 Round Goby shallow and deeper profundal habitat). Due to the sample size differing by one 234 individual, we assumed a balanced design and followed the recommendations of Anderson and 235 Walsh (2013); PERMANOVA remains robust in the presence of heterogeneity of group 236 dispersion.

237 In addition to NMDS, $\beta$-diversity was compared between fish species and habitat using 238 pairwise weighted UniFrac distances (Lozupone and Knight 2005). Unifrac distances were 239 calculated between all combinations of Round Goby and Yellow Bullhead (for the species 240 comparison) and Governor's Island and Eagle Wings Round Goby (for the habitat comparison).

241 Similar to the NMDS ordinations, weighted Unifrac distances were visualized using Principal 242 Coordinate Analysis (PCoA) while retaining the first two axes. Welch's two sample t-tests were 243 conducted to determine if weighted Unifrac distances between sample groups was significant.

244 These distances were subsequently plotted $( \pm \mathrm{SE})$ to visualize differences in gut microbiota by 245 fish species and habitat.

246 Predictions of microbial community functions were assessed using the "Tax4Fun" 247 (Aßhauer et al. 2015) and "themetagenomics" (Woloszynek et al. 2017) R packages. 16S rRNA 248 marker gene functions were linked to SILVA database Kyoto Encyclopedia of Genes and 249 Genomes (KEGG) orthologs (KOs) using the MoP-Pro approach to determine predictive 250 function relative gene abundance for each gut microbiota sample (Aßhauer and Meinicke 2013). 251 The top 20 KO's were then screened (Yang et al. 2019) and plotted on bar graphs to related 252 relative gene abundance vs. fish species or capture location (for Round Goby). Orthologs that did 
253 not link to a specified level one KEGG pathway were excluded from the analyses, and the next 254 most abundant was substituted. Functions were combined based on level one as defined by the 255 KEGG pathways output. Significance testing comparing the gut microbiota samples was 256 performed using Welch's two sample t-tests.

257

258 Supplementary Data Processing and Analysis Information.- All procedures including tissue 259 sampling, DNA extraction, PCR, amplicon cleanup, NGS library preparation, and downstream 260 FASTQ processing as well as all code are detailed on our GitHub page 261 (https://github.com/bngallo1994).

262

263

264

265

266

267

268

269

270

271

272

273

274

275

276

277

278

279

280

281

282

\section{Results}

NGS Sequencing

Species and habitat comparison datasets yielded 3.01 and 1.90 million paired-end sequenced reads, respectively, each passing our pre-defined USEARCH quality filter. Final reads clustered into 1,266 OTUs and 574 OTUs in species and habitat comparison OTU tables, respectively. Rarefaction analyses (Fig. 2A \& 3A) indicated plateauing of OTU detection at approximately 20,000-30,000 reads/sample, but novel OTU's were still detected at upwards of 100,000 reads / sample, especially in Yellow Bullhead. The mean number of reads/sample $\pm \mathrm{SE}$ was $136,959 \pm 776$ with a mean of $126,739 \pm 967$ for Round Goby and $158,858 \pm 1,283$ in Yellow Bullhead. Analysis of rarefaction data using Good's Coverage at the normalized sequencing depth indicated samples represented $>99.7 \%$ coverage of OTU diversity in both datasets (Tables 1 ).

Slope analysis of normalized rarefaction curves indicated high rates of microbe OTU detection at low sequencing depth and low novel OTU detection at higher sequencing depth. Discovery rates of OTUs/1000 sequences show the slope of the normalized rarefaction curves were between 0.091-2.66 OTUs/1000 sequences between both datasets. Further validation of our high OTU coverage was seen comparing the OTU species richness at the normalized sequencing depth to maximum sequencing depth for each sample. The calculated highest sequencing depth provided an average \pm SE increase of $32 \pm 14$ OTUs and $11 \pm 4$ OTUs for the species and habitat datasets respectively (Supplemental Tables S2 \& S3).

283 
284 Comparing Round Goby and Yellow Bullhead Gut Microbiota (SPECIES) and Round Goby

285 Microbiota at the Eagle Wings Islands and Governor's Island (HABITAT)

286 Permutational analysis of variance (PERMANOVA) on the species comparison NMDS

287 ordination (Fig. 2B) indicated a significant difference between Round Goby and Yellow

288 Bullhead gut microbial communities (PERMANOVA: Pseudo $F=7.88, d f=1, P=0.003, r^{2}=$

$2890.28, N M D S$ stress $=0.14$ ). Two distinct groups are evident with $95 \%$ confidence ellipses

290 displaying little overlap (Fig. 2B). Similar patterns were observed with the weighted Unifrac

291 PCoA ordination (Axes $1+2$ accounting for over $73 \%$ of total data variation (Supplemental Fig 292 S3)).

293 OTU identification in both Round Goby and Yellow Bullhead also showed differences in 294 the bacterial composition of dominant microbes in each of the fish species. Parsing the present 295 microbiota by relative abundance, the top 10 OTUs (Fig. 4) using the SILVA database showed 296 Aeromonas spp. (OTU1, 66.9 \pm 8.7\%), Cetobacterium spp. (OTU2, $10.1 \pm 6.4 \%$ ), and 297 Streptococcus spp. (OTU5, $4.4 \pm 3.3 \%$ ), as the most abundant taxa in the gut microbiota of 298 Round Goby. While Cetobacterium spp. (OTU2, 39.7士 14.7\%), Clostridium sensu stricto 1 299 (OTU7, 25.7 $\pm 8.7 \%$ and Aeromonas spp. (OTU1, 14.8 \pm 9.4\%) were the most abundant bacteria 300 in Yellow Bullhead.

301

Similar analyses comparing Round Goby sampled between Governors Island and the

302 Eagle Wings Islands (Fig. 3B) also revealed differences in their gut microbial composition 303 (PERMANOVA: Pseudo $F=2.63, d f=1, P=0.03, r^{2}=0.17, N M D S$ stress $=0.04$ ). PCoA 304 ordinations showed similarity to the NMDS plot (supplemental Figure S4), and the plotted 305 principal components accounted for over $89 \%$ of the data's variation. Additionally, differences 306 307 were observed in the dominant gut microbiota from Round Goby at each location (Fig. 5). The Eagle Wing's Round Goby gut samples were dominated by Aeromonas spp. (OTU1, $88.9 \pm$ 308 $4.0 \%$ ), Shewanella spp. (OTU8, $4.2 \pm 2.7 \%$ ), and Corynebacterium spp. (OTU14, $1.1 \pm 0.7 \%$ ) while Governors Island Round Goby guts were largely colonized by Aeromonas spp. (OTU1, $47.3 \pm 12.4 \%$ ), Cetobacterium spp. (OTU2, $18.6 \pm 11.6 \%$ ) and Streptococcus spp. (OTU3, $8.1 \pm$ $6.1 \%$ ) bacteria. In both comparisons, the Top 10 dominant bacteria accounted for over $89 \%$ of 312 the total microbiota observed.

Despite the observed differences in the dominant taxa between and within sampled fish gut microbiota, no significant differences existed for any $\alpha$-diversity metrics, including the 
315 Observed, Chao1, Shannon, and Simpson indices (P-values > 0.18) among species (Fig. 6).

316 Similar trends were seen within Round Goby through the habitat comparison (P-values $>0.23$;

317 Fig. 7). UniFrac distances however, revealed higher variance in the gut bacterial community

318 between species (RG: $t=-4.11, d f=293.37, P<0.001 \&$ YB: $t=3.20, d f=84.47, P=0.002$ )

319 compared to within species (Fig. 8). For Round Goby, additional Unifrac distance testing

320 indicated a significant difference in bacterial community variation between habitats $(t=11.30 \mathrm{df}$

$321=50.49, P<0.001)$. Round Goby from the deepwater habitat (Eagle Wings Islands)

322 demonstrated the least amount of variation in gut microbial composition while those from the

323 nearshore littoral Governors Island had the greatest amount of variation (Fig. 9).

324

325 Comparing Round Goby and Yellow Bullhead Gut Microbiota Predicted Functions using

326 Tax4Fun

327 Predictions of microbial community functions using KEGG Orthologs (KO) indicated no

328 significant differences in predicted microbial functions associated with our species and habitat

329 comparisons. Mapping of the Top 20 KO's that matched to KEGG pathways revealed only slight

330 differences in microbial community gene abundances linked with environmental information

331 processing, cellular processes, metabolic pathways, and genetic information processing (Fig. 10

$332+11)$. There were no significant differences in the top $20 \mathrm{KO}$ 's for the species comparison for

333 environmental information processing $(t=0.28, d f=9.22, P=0.79)$, cellular processes $(t=0.18$,

$334 d f=10.07, P=0.86)$, metabolic pathways $(t=0.58, d f=19.75, P=0.57)$, and genetic

335 information processing $(t=-0.19, d f=9.36, P=0.86)$. Similar conclusions were drawn from

336 analysis of the top $20 \mathrm{KO}$ 's for the habitat comparison for Round Goby (environmental

337 information processing: $t=-1.86, d f=7.00, P=0.11$; cellular processes: $t=0.18, d f=10.07, P=$

338 0.86; metabolic pathways: $t=1.93, d f=7.13, P=0.09$; genetic information processing: $t=1.75$,

$339 d f=7.05, P=0.12$ ). This all stands in contrast to the aforementioned significant differences in

340 overall gut microbiota both between and within species as seen through our $\beta$-diversity analyses.

341

342 Discussion.

343 Our data revealed fish gut microbiota differences between invasive Round Goby and

344 native Yellow Bullhead in the upper St. Lawrence River. Our findings add to previous research

345 that describes microbial community dissimilarities in various fish species employing a range of 
346 sequencing methods (Denaturing Grade Gel Electrophoresis (Li et al. 2012); Pyrosequencing (Li 347 et al. 2014)) as well as amplifying different variable regions of the 16S rRNA gene (V4 region;

348 Liu et al. 2016). Both NMDS and PCoA analyses support overall microbial composition

349 differences between these fish species and results from the SILVA database indicated dominance

350 of specific bacterial genera in each species' gut microbiome. Additional hypothesis testing using 351 PERMANOVAs and weighted Unifrac distances further verified that significant differences in $\beta$ 352 diversity were present both between Round Goby and Yellow Bullhead, and within Round Goby 353 caught at the Eagle Wings and Governor's Island.

The observed prominence of Aeromonas spp., Cetobacterium spp., and Clostridium spp.

355

356

357

358

359

360

361

362

363

364

365

366

367

368

369

370

371

372

373

374

375

376

in the fish gut is consistent with reports by others including Aeromonas spp. (Wu et al. 2013; Li et al. 2016), Cetobacterium spp. (Li et. al 2015), and Aeromonas spp. and Cetobacterium somerae (Larsen et al. 2014). In addition to microbial differences noted between fish species, we also report interspecies variation in gut microbiota between the same species (Round Goby) captured in different habitats separated by a water depth gradient. Simulation analysis from Anderson and Walsh (2013) suggests PERMANOVA analysis is robust even with heterogeneity of variance between groups under a balanced design. Larger scale sampling from multiple locations with a balanced sampling design is recommended to better understand patterns of within species differences in microbiome among habitats. Future studies should take into account size and/or age bias with Round Goby, given the differences in average size of the captured Round Goby by habitat (150mm at Governor's Island vs. $113 \mathrm{~mm}$ at the Eagle Wings). Previous research on Zebrafish has indicated that gut microbiota can vary with age/development (Stephens et al. 2016) and by seasonal sampling (Naviner et al. 2006). All fish associated with this study were captured within an 11-day period during the fall of 2017, however observed differences in gut microbiota may not be present throughout all seasons. These variables will need to be controlled in future studies that increase sampling frequency spatiotemporally to extend our finding in a simple case.

The presence of specific and novel OTUs is particularly interesting when hypothesizing the microbiota's role in the gut community and its effect on fish hosts. For example, some species of Aeromonas act as opportunistic pathogens and cause various hemorrhagic fish diseases like furunculosis (Hidalgo et al. 2012). Although Aeromonas spp. were routinely detected in fish guts in this study, we observed no obvious tissue hemorrhaging. However, we

Peer] reviewing PDF | (2020:02:45747:2:0:NEW 24 Sep 2020) 
377 were unable to resolve Aeromonas sequences to the species level and were not able to determine

378 if the specific Aeromonas bacteria present were known fish pathogens. This was most likely due

379 to high heterogeneity of the 16S gene in Aeromonas bacteria (Janda and Abbott 2007).

380 Sequencing with additional universal gene primers (e.g., cpn60 (Miñana-Galbis et al. 2009) may

381 help reveal species delineation in Aeromonas bacteria. Additional microbial genera detected in

382 this study are also known to be beneficial and/or detrimental to their hosts. For example, bacteria

383 of the genera Clostridium produce various essential fatty acids and vitamins (Ringø et al. 1995;

384 Givens 2014) despite some species like Clostridium difficile, which can opportunistically cause

385 pseudomembranous colitis (Kelly et al. 1994). Moreover, the bacterium Cetobacterium somerae,

386 the most abundant genera discovered in the gut microbiome of Yellow Bullhead in our study, is

387 known to synthesize vitamin B12 in the fish gut (Tsuchiya et al. 2007). Regardless, 16S

388 predictive functions derived from our Tax4Fun analyses were unable to identify microbiota

389 functional patterns between Round Goby and Yellow Bullhead and within Round Goby by

390 sampled habitat. In order to gain a better understanding of potential gut microbiota function in

391 the fish gut, sampling with shotgun sequence metagenomic analyses could be implemented with

392 parallel 16S amplicon sequencing analysis. The 16S amplicon sequencing would help delineate

393 relative abundance of microbiota, and metagenomic analysis would define individual microbes'

394 complete genomes. Together these approaches may provide a more complete picture regarding

395 the metabolic, pathogenic, and other characteristics associated with bacteria sampled from the

396 fish gut. The need for metagenomic analysis in has been called for in recent reviews on the fish

397 gut microbiome (Tarnecki et al. 2017; Gallo et al. 2020) and implementation will almost

398 certainly provide a better understanding on gut microbial functions that may significantly

399 influence fish health.

400 In addition to the demonstration of microbial differences across species, NGS has the

401 potential to detect minute differences in microbial communities within species. We employed an

402 NGS workflow to investigate differences in gut microbiota in Round Goby samples from

403 different habitats separated by less than 750 meters. Evidence for environmental differences in

404 fish gut microbiota has been previously noted in the Atlantic salmon parr gut microbiome

405 (Dehler et al. 2017) and in wild vs. aquaculture-reared fine flounder (Ramírez and Romero

406 2017). Although our design did not take into account potential diet and habitat variation between

407 the two samples of Round Goby, our data does support within-species differences at fine-spatial 
408 scales. A previous study in a nearby area of the St. Lawrence River indicated significant 409 differences in total phosphorus and crustacean zooplankton abundance between shallow and 410 deepwater stations (Farrell et al. 2010). These differences possibly extend to benthic 411 invertebrates, including dressenid mussels known as important forage for Round Goby. Both 412 Round Goby habitats were sampled at different depths (Governors Island: <2m; Eagle Wings 413 Islands: $>15 \mathrm{~m}$ ) and significant gut microbial community differences were noted, specifically 414 with Aeromonas spp. (>80\% total Eagle Wings Islands reads vs. $~ 50 \%$ of Governors Island 415 reads). Nonetheless, $\alpha$-diversity metrics indicated no differences in gut microbiota abundance in 416 both our habitat and species comparisons. These analyses support that despite overall differences 417 in the taxonomy of the bacteria, the diversity and evenness of the microbial communities within 418 compared groups remained similar. The similarity in $\alpha$-diversity metrics especially within the 419 species comparison may point to limited ecological niches for microbiota to inhabit in the fish 420 gut. Therefore even though the microbiota identity differs between species, Bacteria having 421 similar metabolic capacities / functions in the gut environment prevent large fluctuations in $\alpha$ 422 diversity between fish species. Future analysis derived from NGS microbial data coupled with 423 biological (e.g., host diet, host habitat) and environmental factors (e.g., pressure, temperature, 424 and light penetration) would help further define gut community structure differences observed 425 between habitats and fish species.

426

\section{Conclusions}

In this study, we employed an NGS workflow and sampled gut microbiota for the first time from fishes along the upper St. Lawrence River. We implement analyses to test both interand intraspecific differences. We describe significant species level microbial community differences between an invasive (Round Goby) and native (Yellow Bullhead) fish species. We also describe significant differences between Round Goby sampled in different habits separated by only $\sim 750$ meters. This detailed workflow may help facilitate greater application of gut microbiome research and allow for the examination of numerous basic and applied questions (for examples see Gallo et al. 2020). As NGS technologies and knowledge of host-microbe interactions continue to grow, investigations into the gut microbiome will undoubtedly improve our understanding of fish ecology and their conservation and management. The research methods 
438 developed and applied here are intended to promote such investigations, and aid researchers 439 interested in studying gut microbiota.

440

441 Acknowledgements

442 A special thanks is given to the managers, postdocs, fellow graduate students, and

443 technicians at TIBS that aided in collection of fish samples. We would also like to thank Dr.

444 Donald Walker from Middle Tennessee State University and Dr. Karine Leydet from Syracuse

445 University for providing a review prior to submission. This research is a contribution of the

446 Thousand Islands Biological Station.

447

448

449

450

451

452

453

454

455

456

457

458

459

460

461

462

463

464

465

466

467

468

469

470

471 


\section{References}

473 Aßhauer, K. P., and Meinicke, P. 2013. On the estimation of metabolic profiles in metagenomics.

474 (Göttingen: Schloss Dagstuhl - Leigniz-Zentrum für Informatik GmbH), 1-13.

475

476 Aßhauer, K. P., Wemheuer, B., Daniel, R., and Meinicke, P. 2015. Tax4Fun: predicting

477 functional profiles from metagenomics 16S rRNA data. Bioinformatics 31(17): 2882-2884. doi:

478 10.1093/bioinformatics/btv287.

479

480

Anderson, M. J, and Walsh, D. C. I. 2013. PERMANOVA, ANOSIM, and the Mantel Test in the 481 face of heterogenous dispersions: What null hypothesis are you testing? Ecological Monographs 482 83(4): 557-574. Doi: 10.1890/12-2010.1

483

484

Bray, J. R., and J. T. Curtis. 1957. An ordination of the upland forest communities of southern 485 Wisconsin. Ecological Monographs 27(4): 325-349.

486

487

Brown, S. P., A. M. Veach, A. R. Rigdon-Huss, K. Grond, S. K. Lickteig, K. Lothamer, A. K. 488 Oliver, and A. Jumpponen. 2015. Scraping the bottom of the barrel: are rare high throughput 489 sequenes artifacts? Fungal Ecology 13:221-225.

490

491 Camacho-Sanchez, M., P. Burraco, I. Gomez-Mestre, and J. A. Leonard. 2013. Preservation of 492 RNA and DNA from mammal samples under field conditions. Molecular Ecology Resources 493 13(4): 663-673.

494

495

Chao, A. 1984. Nonparametric estimation of the number of classes in a population. Scandinavian Journal of Statistics 11(4): 265-270.

497

498

Chao, A., L. Jost. 2012. Coverage-based rarefaction and extrapolation: standardizing samples by 499 completeness rather than size. Ecology 93(12): 2533-2547.

500

501 Charlebois, P. M., L. D. Corkum, D. J. Jude, and C. Knight. 2001. The Round Goby (Neogobius 502 melanstomus) invasion: current research and future needs. Journal of Great Lakes Research 
503 27(3): 263-266.

504

505 Colombo, B. M., T. Scalvenzi, S. Benlamara, and N. Pollet. 2015. Microbiota and mucosal

506 immunity in amphibians. Frontiers in Immunology 6(11): 1-111.

507

508 Comeau, A. M., W. K. W. Li, J-É Tremblay, E. C. Carmack, and C. Lovejoy. 2011. Artic Ocean 509 microbial community structure before and after the 2007 record sea ice minimum. PLoS ONE 510 6(11): e27492.

511

512 Comeau, A. M., G. M. Douglas, and M. G. I. Langille. 2017. Microbiome Helper: a custom and 513 streamlined workflow for microbiome research. mSystems 2(1): e0127-16.

514

515 Dehler, C., C. Secombes, and S. Martin. 2017. Environmental and physiological factors shape 516 the gut microbiota of Atlantic Salmon parr (Salmo salar L.) Aquaculture 467: 149-157.

517

518 Edgar, R., C. 2013. UPARSE: highly accurate OTU sequences from microbial amplicon reads.

519 National Methods 10: 996-998.

520

521 Eichmiller, J. J., M. J. Hamilton, C. Staley, M. J. Sadowsky, and P. W. Sorensen. 2016.

522 Environment shapes the fecal microbiome of invasive carp species. Microbiome 4(1):44.

523

524 Farrell, J. M., K. T. Holeck, E. L. Mills, C. E. Hoffman, and V. J. Patil. 2010. Recent ecological

525 trends in lower trophic levels of the International Section of the St. Lawrence River: A

526 comparison of the 1970s to the 2000s. Hydrobiologia 647:21-33.

527

528

529 Gallo, B., J. M. Farrell, and B. Leydet. 2020. Fisheries and the gut microbiome: using next

530 generation sequencing (NGS) and microbial ecology to better understand fish-microbe

531 interactions. Fisheries 45(5): 271-282.

532

533 Good, I. J. 1953. The populatioon frequencies of species and the estimation of population 
534 parameters. Biometrika 40(3-4): 237-264.

535

536 Ghanbari, M., W. Kneifel, and K. J. Domig. 2015. A new view of the figh gut microbiome:

537 Advances from next-generation sequencing. Aquaculture 448: 464-475.

538

539 Givens, C. E. 2014. A fish tale: comparison of the gut microbiome of 15 fish species and the

540 influence of diet and temperature on Its composition. PhD Dissertation. University of Georgia,

541 Athens, GA. doi: 10.1371/journal.pone.0166379.

542

543 Hennersdorf, P., S. Kleinertz, S. Theisen, M. A. Abdul-Aziz, G. Mrotzek, H. W. Palm, and H. P.

544 Saluz. 2016. Microbial diversity and parasitic load in tropcial fish of different environmnetal

545 conditions. PLoS ONE 11(3): e0151594.

546

547 Hidalgo, R. B., and M. J. Figueras. 2012. Molecular detection and characterization of

548 furunculosis and Other Aeromonas fish infections in Health and environment in aquaculture. Dr.

549 Edmir Carvalho, editor. ISBN: 978-953-51-0497-1, InTech, Available from:

550 http://cdn.intechopen.com/pdfs/35139/InTechMolecular detection and characterization of furu

551 nculosis and other aeromonas fish infections.pdf.

552

553 Hurlbert, S. H., 1971. The nonconcept of species diversity: a critique and alternative parameters.

554 Ecology 52(4): 577-586.

555

556 Janda, J. M., and S. L. Abbott. 2007. 16S rRNA gene sequencing for bacterial identification in

557 the diagnostic laboratory: pluses, perils, and pitfalls. Journal of Clinical Microbiology 45(9):

558 2761-2764.

559

560 Johnson, K. V.-A., and K. R. Foster. 2018. Why does the microbiome affect behavior? Nature

561 Reviews: Microbiology 16(10): 647-655.

562

563 Kelly, C. P., C. Pothoulakis, and J. T. LaMont. 1994. Clostridium difficile Colitis. The New

564 England Journal of Medicine 330: 257-262. 
565

566 Kruskal, J. B. 1964. Multidimensional scaling by optimizing goodness of fit to a nonmetric 567 hypothesis. Psychometrika 29(1): 1-27.

568

569

Larsen, A. M., H. H. Mohammed, and C. R. Arias. 2014. Characterization of the gut microbiota 570 of three commercially valuable warmwater fish species. Journal of Applied Microbiology 571 116(6): 1396-1404.

572

573 Li, J., J. Ni, J. Li, C. Wang, X. Li, S. Wu, T. Zhang, Y. Yu, and Q. Yan. 2014. Comparative 574 study on gastrointestinal microbiota of eight fish species with different feeding habits. Journal of 575 Applied Microbiology 117(6): 1750-1760.

576

577 Li, T., Long, M., Gatesoupe, F-J., Zhang, Q., Li, A., and Gong, X. 2015. Comparative Analysis 578 of the Intestinal Bacterial Communities in Different Species of Carp by Pyrosequencing.

579 Microbal Ecology 69:25-36. doi: 10.1007/s00248-014-0480-8.

580

581

Li, T., M. Long, C. Ji, Z. Shen, F-J, Gatesoupe, X. Zhang, Q. Zhang, L. Zhang, Y. Zhao, X. Liu, 582 and A. Li. 2016. Alterations of the gut microbiome of Largemouth Bronze Gudgeon (Coreius 583 guichenoti) suffering from furunculosis. Scientific Reports 6: 30606.

584

585 Li, X., Y. Yu, W. Feng, Q. Yan, and Y. Gong. 2012. Host species as a strong determinat of the 586 587

Liu, H., X. Guo, R. Gooneratne, R. Lai, C. Zeng, F. Zhan, and W. Wang. 2016. The gut 589 microbiome and degradation enzyme activity of wild freshwater fishes influenced by their 590 trophic levels. Scientific Reports 6 (24340).

591

592

Lozupone, C. A., Knight, R. 2005. Unifrac: a New Phylogenetic Method for Comparing 593 Microbial Communities. Appied Environmental Microbiology. 71(12): 8228-8235. 
596 stability and resilience of the human gut microbiota. Nature 489: 220-230.

597

598 Marcon, E. 2018. 'Entropart v1.5-3' - Entropy Partioning to Measure Diversity. Available at

599 https://EricMarcon.github.io/entropart.

600

601 Madden, T. 2013. The BLAST sequences analysis tool In The NCBI Handbook [Internet]. $2^{\text {nd }}$

602 edition.National Center for Biotechnology Information, Bethesda, Maryland. Available from:

603 https://www.ncbi.nlm.nih.gov/books/NBK153387/.

604

605 McMurdie, P. J., and Holmes, S. 2013. phyloseq: An R package for reproducible interactive 606 analysis and graphics of microbiome census data. PLoS ONE 8(4), e61217.

607

608

Menke, S., Gillingham, M. A. F., Wilhelm, K., and Sommer, S. 2017. Home-Made Cost

609

Effective Preservation Buffer Is a Better Alternative to Commercvial Preservation Methods for

610

Microbiome Research. Frontiers in Microbiology 8:102.

611

612 Michl, S. C., J-M., Ratten, M. Beyer, M. Hasler, J. LaRoche, and C. Schulz, 2017. The malleable

613 gut microbiome of juvenile Rainbow Trout (Oncorhynchus mykiss): diet-dependent shifts of

614 bacterial community structures. PLoS ONE 12(5): e0177735.

615

616 Miñana-Galbis, D., A.Urbizu-Serrano, M. Farfán, M. C. Fusté, and J. G. Lorén. 2009.

617 Phylogenetic analysis and identification of Aeromonas species based on sequencing of the cpn60

618 universal target. International Journal of Systematic and Evolutionary Microbiology 59: 1976-

6191983.

620

621 Oksanen, J. F., F. G. Blanchet, M. Friendly, R., Kindt, P. Legendre, D. McGlinn, P. R. Minchin,

622 R. B. O’Hara, G. L. Simpson, P. Solymos, M. H. M. Stevens, E. Szoecs, and H. Wagner. 2018.

623 Vegan: community ecology package. R package version 2.5.2. Available at https://CRAN.R-

624 project.org/package=vegan.

625

626 Benjamini, Y., and Hochberg, Y. 1995. Controlling the False Discovery Rate: A Practical and 
627 Powerful Approach to Multiple Testing. Journal of the Royal Statistical Society. Series B

628 (Methodological). 57(1): 289-300.

629

630 Pruesse, E., Peplies, J., and Glöckner, F. O. 2012. SINA: accurate high-throughput multiple 631 sequence alignment of ribosomal RNA genes. Bioinformatics 28: 1823-1829.

632

633 Quast, C., Pruesse, E., Yilmaz, P., Gerken, J., Schweer, T., Yarza, P., Peplies, J., and Glöckner, 634 F. O. 2013. The SILVA Ribosomal RNA Gene Database Project: Improved Data Processing and 635 Web-Based Tools. Nuclei Acids Research 41(D1): D590-D596.

636

637 R Core Team. 2017. R: a language and environment for statistical computing. https://www.R638 project.org/.

639

640 Ramírez, C., and J. Romero. 2017. Fine Flounder (Paralichthys adspersus) microbiome showed 641 important differences between wild and reared specimens. Frontiers in Microbiology 8:271.

642

643 Ray, W. J., and L. D. Corkum. 1997. Predation of Zebra Mussels by Round Gobies, Neogobius 644 melanostomus. Environmental Biology of Fishes 50(3): 267-273.

645

646 Naviner, M., Giraud, E., Le Bris, H., Armand, F., Mangion, C., and Ganière, J-P. 2006. Seasonal 647 variability of intestinal microbiota in rainbow trout (Oncorhynchus mykiss), with a particular 648 attention to Aeromonas spp. as candidate indicator of antimicrobial resistance. Revue de 649 medicine vétérinaire 157(12): 599-604.

650

651 Ringø, E., E. Strøm, and J-A, Tabachek. 1995. Intestinal microflora of salmonids: a review.

652 Aquaculture Research 26(10): 773-789.

653

654 Roeselers, G., E. K. Mittge, W. Z. Stephens, D. M. Parichym, C. M. Cavanaugh, K.

655 Guilleminm, and J. F. Rawls. 2011. Evidence for a core gut microbiota in the Zebrafish. The

656 ISME Journal 5(10): 1595-1608.

657 
658 Schloss, P.D., S. L. Westcott, T. Ryabin, J. R. Hall, M. Hartmann, E. B. Hollister, R. A.

659 Lesniewski, B. B. Oakley, D. H. Parks, C. J. Robinson, J. W. Sahl, B. Stres, G. G. Thallinger, D.

660 J. Van Horn, and C. F. Weber. 2009. Introducing mothur: open-source, platform-independent,

661 community-supported software for describing and comparing microbial communities. Applied

662 Environmental Microbiology 75(23): 7537-41.

663

664 Schmidt, V. T., Smith, K. F., Melvin, D. W., and Amaral-Zettler, L. A. 2015. Community

665 assembly of a euryhaline fish microbiome during salinity acclimation. Molecular Ecology 24(10:

$666 \quad 2537-2550$.

667

668 Stegemann, E. C. 1989. Some catfishes of New York. The Conservationist 44(1) 40-45.

669 Available from http://nysl.cloudapp.net/awweb/main.jsp?flag=browse\&smd=2\&awdid=4.

670

671 Stephens, W. Z., A. R. Burns, K. Stagaman, S. Wong, J. F. Rawls, K. Guillemin, and B. J. M.

672 Bohannan. 2016. The composition of the Zebrafish intestinal microbial community varies across 673 development. The ISME Journal. 10:644-654.

674

675 Sullam, K. E., S. D. Essinger, C. A. Lozupone, M. P. O’Connor, G. L. Rosen, R. Knight, S. S.

676 Kilham, and J. A. Russell. 2012. Environmental and ecological factors that shape the gut

677 bacterial communities of fish: a meta-analysis. Molecular Ecology 21(13).

678

679 Tarnecki, A. M., F. A. Burgos, C. L. Ray, and C. R. Arias. 2017. Fish intestinal microbiome:

680 diversity and symbiosis unravelled by metagenomics. Journal of Applied Microbiology 123(1),

$6812-17$.

682

683 Tsuchiya, C., T. Sakata, and H. Sugita. 2007. Novel ecological niche of Cetobacterium somerae, 684 an anaerobic bacterium in the intestinal tracts of freshwater fish. Letters in Applied Microbiology 685 46(2): 282.

686

687 Wickham, H., 2016. ggplot2: elegant graphics for data analysis. Springer-Verlag, New York.

688 Version 3.0.0. Available at http://ggplot2.org. 
689

690 Woloszynek, S., Mell, J. C., Zhao, Z., Simpson, G., O’Connor, M. P., and Rosen, G. L. 2019.

691 Exploring thematic structure and predicted functionality of 16S rRNA amplicon data. PLoS ONE 692 14(12): e0219235.

693

694 Wu, S., G. Wang, E. R. Angert, W. Wang, W. Li, and H. Zou. 2012. Composition, diversity, and 695 origin of the bacterial community in Grass Carp intestine. PLoS ONE 7(2): e30440. 
Figure 1

Sampling locations along the St. Lawrence River (Clayton, NY).

Round Goby were sampled at Governors Island (blue: RG30-RG45) and the Eagle Wings Islands (white: RG25-RG29). Yellow Bullhead were sampled at French Creek (red: YBH39YBH56). Circles denote approximate sample location (Image generated using ggmap: Kahle \& Wickham, 2013). Map credit: (c) 2019 Google.

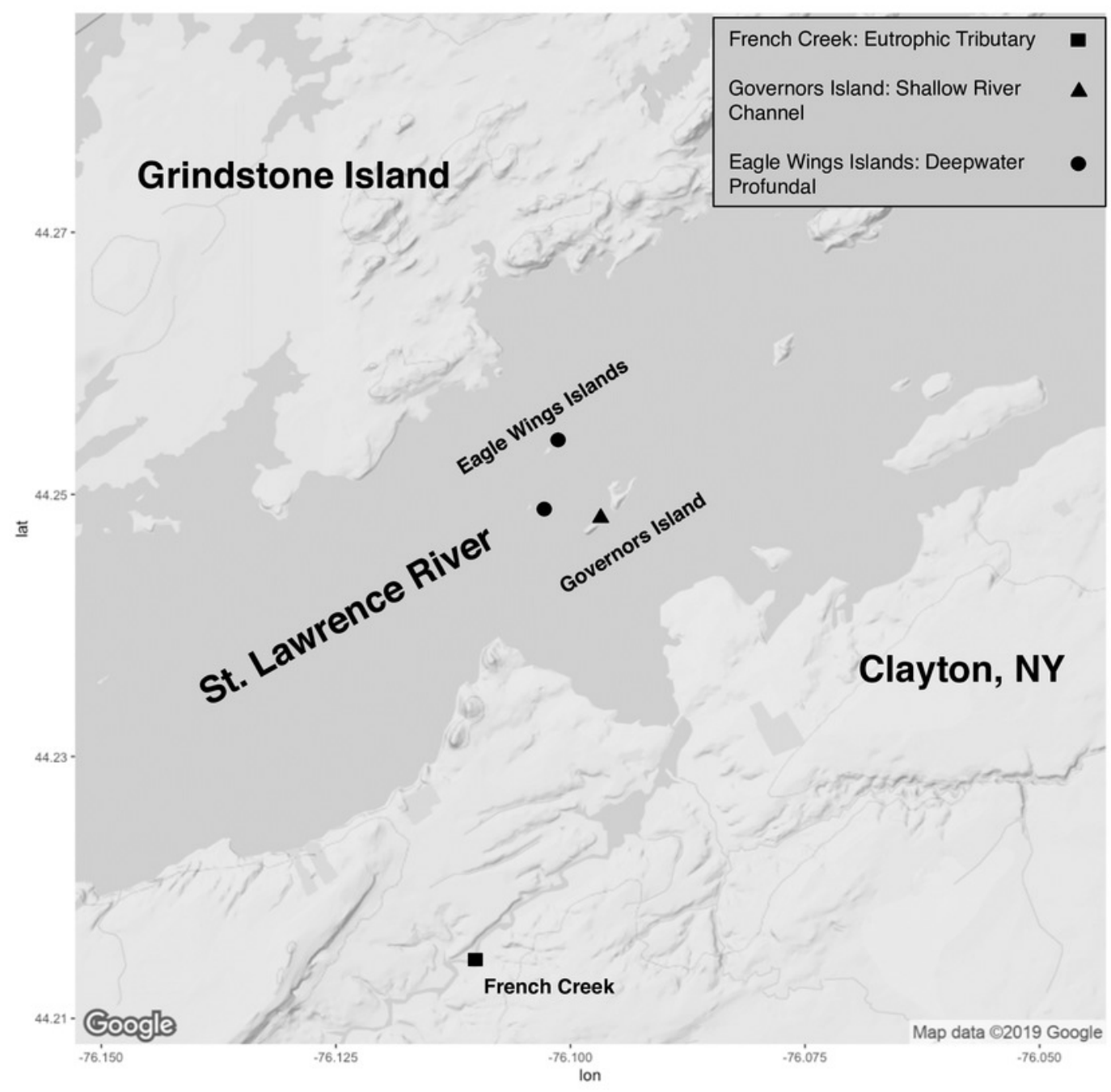


Figure 2

Rarefaction Curve (A) and NMDS ordination (B) for species comparison between Round Goby (RG) and Yellow Bullhead (YBH).

Ellipses on the NMDS plot denote $95 \%$ confidence intervals. The NMDS ordination revealed a significant difference $(P=0.003)$ between the two species.
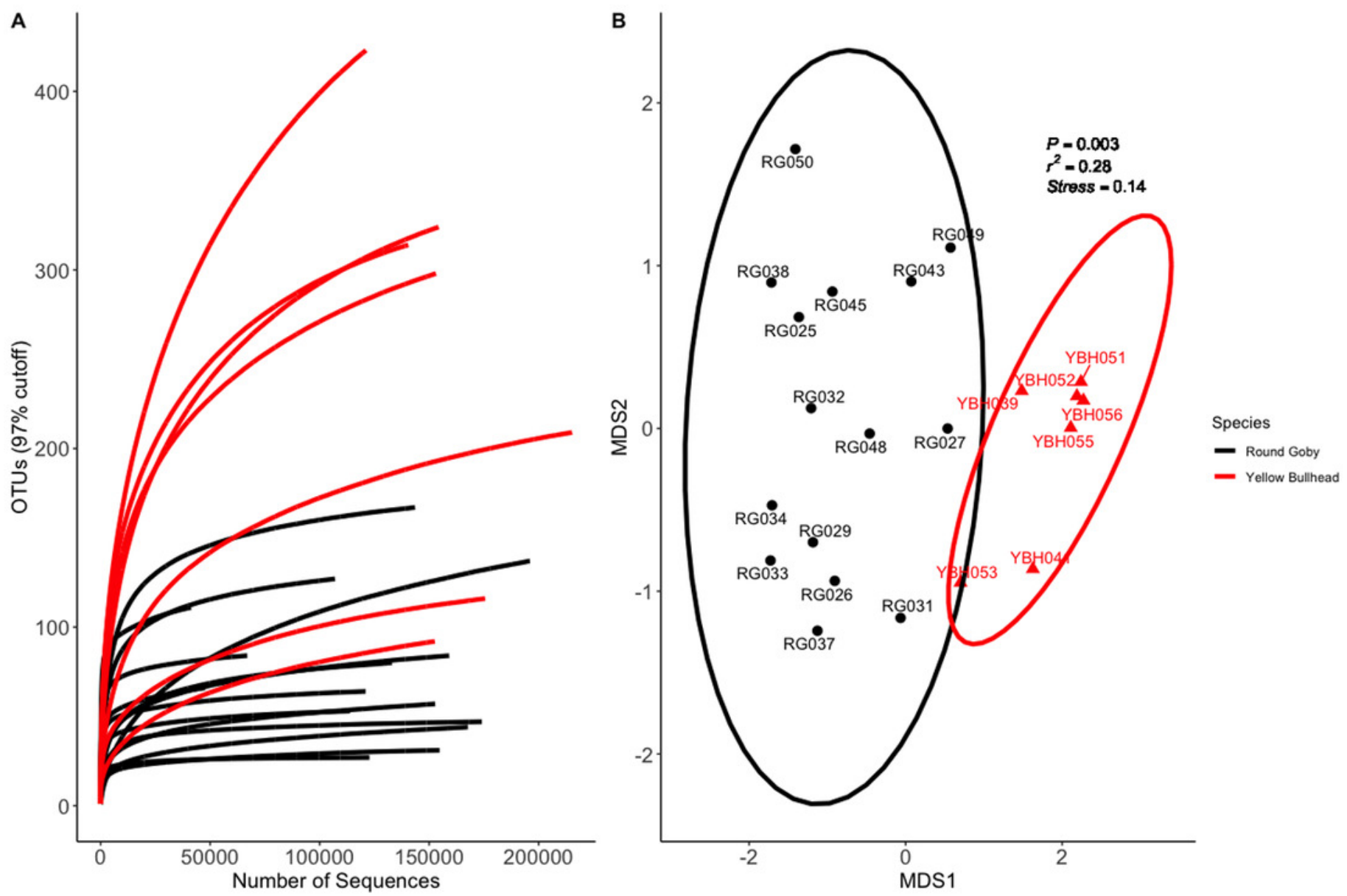
Figure 3

Rarefaction Curve (A) and NMDS plot (B) for Round Goby (RG) habitat comparison between Governors Island and the Eagle Wings Islands.

Ellipses on the NMDS plot denote $95 \%$ confidence intervals. A significant difference $(P=$ 0.03) was found between the Round Goby at each respective location.
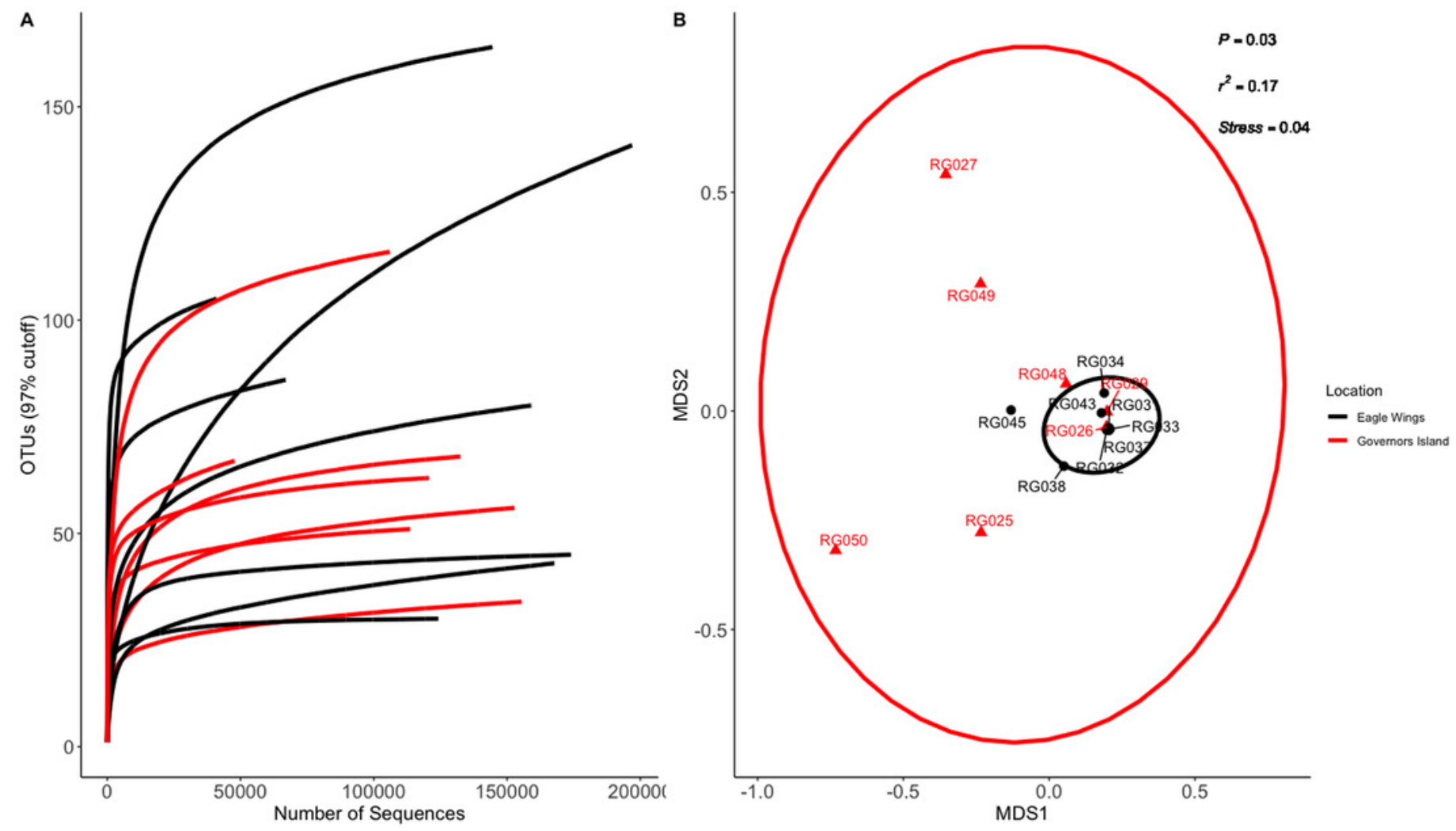


\section{Figure 4}

Relative abundance of Top 10 OTU's for Round Goby and Yellow Bullhead from the species comparison.

Aeromonas spp., Cetobacterium spp., and Streptococcus spp. were the most abundant genera in Round Goby, while Cetobacterium spp., Clostridium sensu stricto 1, and Aeromonas were most abundant in Yellow Bullhead.

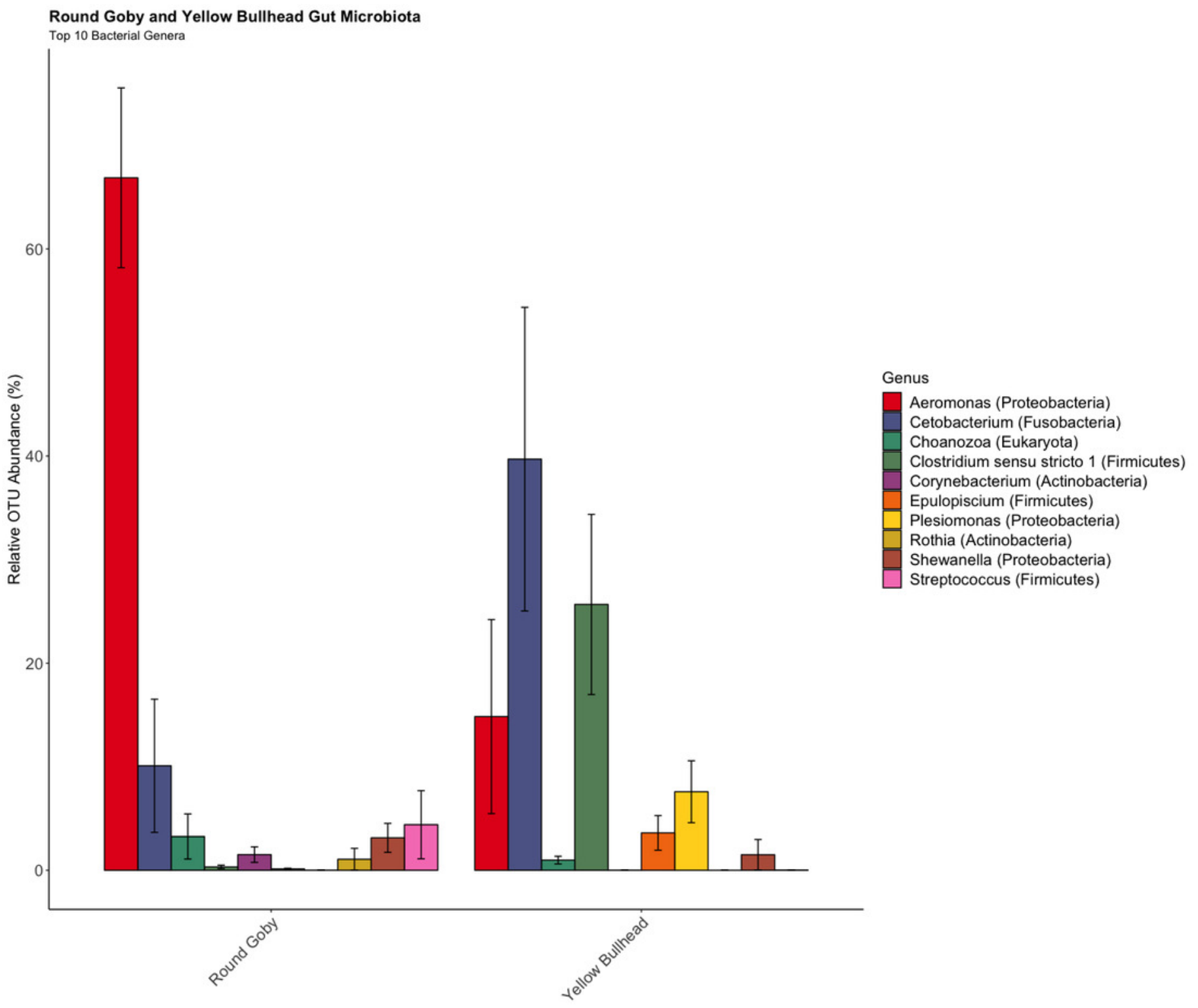




\section{Figure 5}

Relative abundance of Top 10 OTU's for Round Goby by location from the habitat comparison.

Aeromonas spp. dominated the gut microbiota of Round Goby at the Eagle Wing's, while the gut microbiota of Governor's Island Goby was spread out across multiple genera including Aeromonas spp., Cetobacterium spp., and Streptococcus spp.

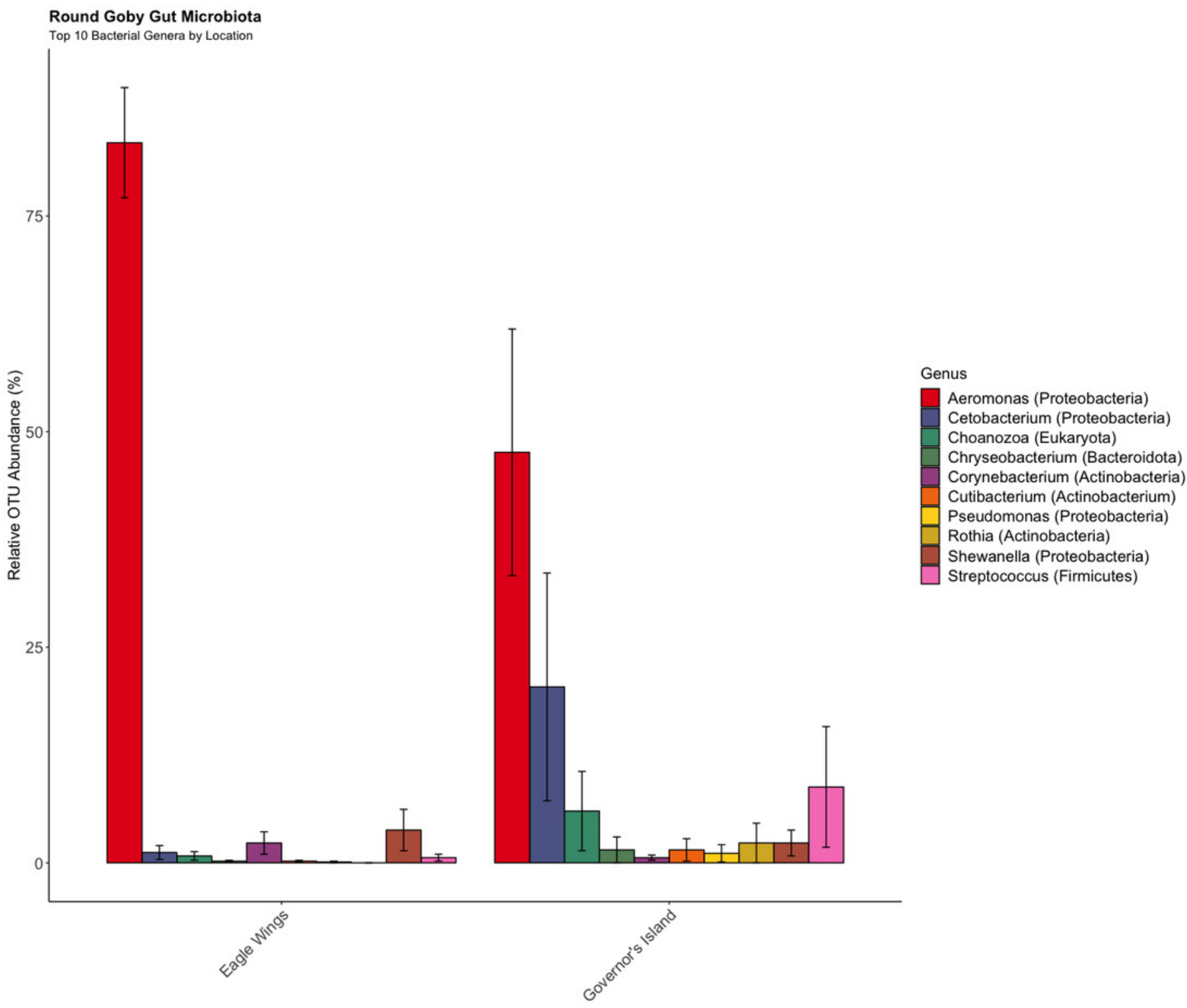


Figure 6

$\alpha$-diversity metrics calculated from the Round Goby vs. Yellow Bullhead species comparison.

(A) Observed, (B) Chaol, and (C) Shannon $\alpha$-diversity incidences showed no differences between species when tested via Wilcoxon rank-sum tests (Mann-Whitney) (all P-values > $0.18)$. 


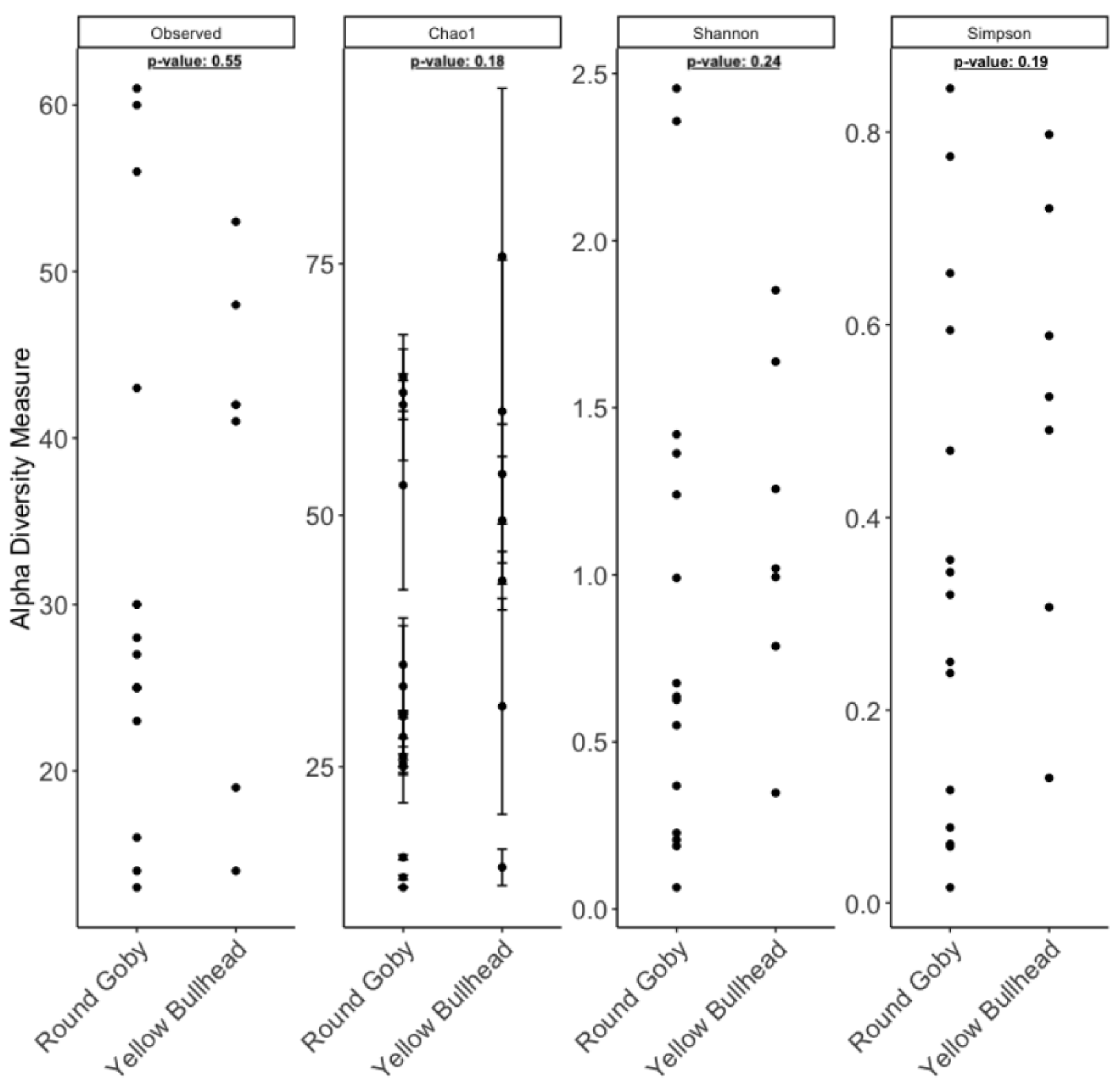

Species 
Figure 7

$\alpha$-diversity metrics calculated from the Round Goby habitat comparison.

(A) Observed, (B) Chaol, and (C) Shannon $\alpha$-diversity incidences showed no differences between habitats when tested via Wilcoxon rank-sum tests (Mann-Whitney) (all P-values > $0.46)$. 


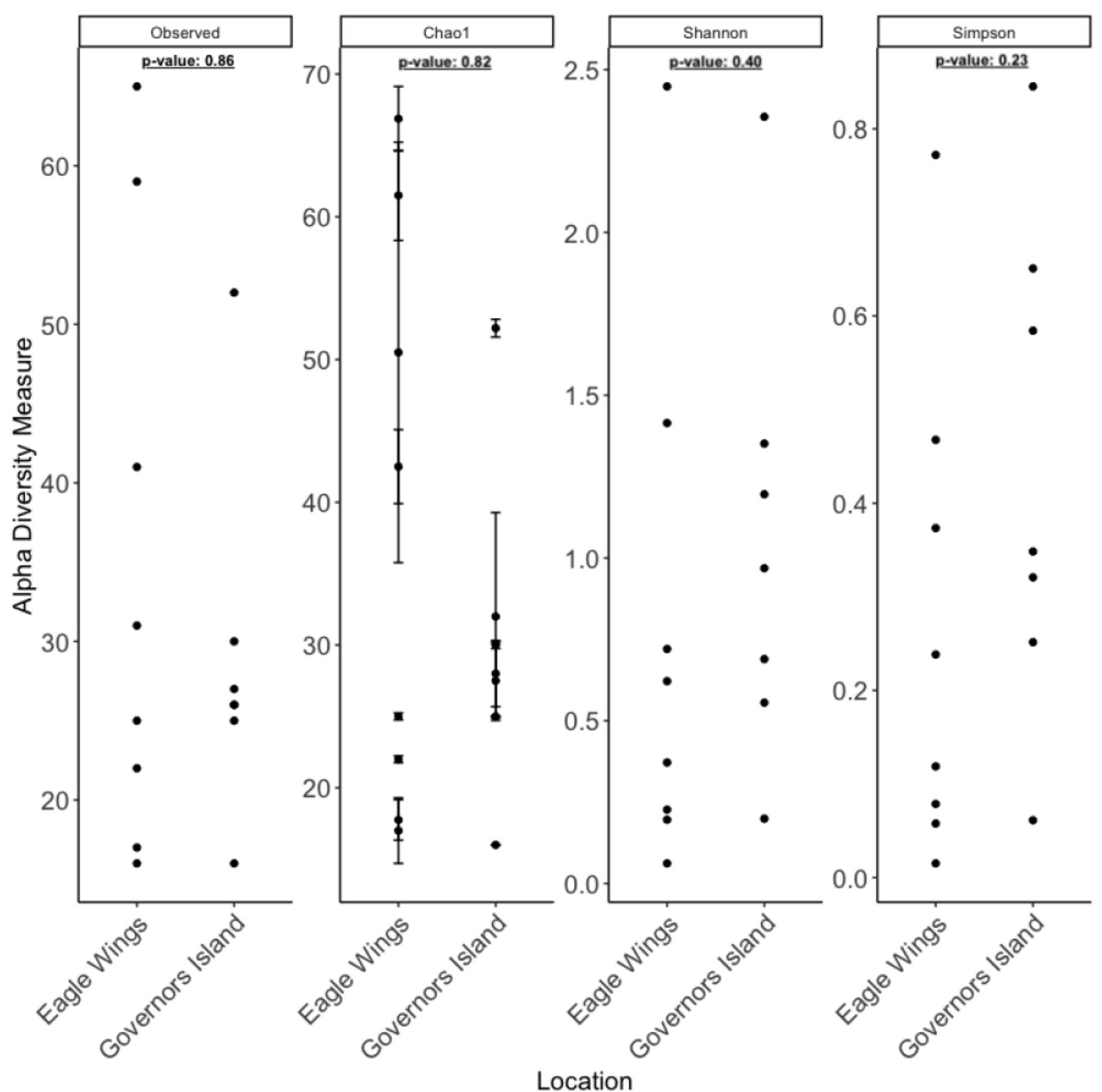




\section{Figure 8}

Pairwise UniFrac distances calculated from the OTU's derived from the species comparison.

The UniFrac analysis revealed higher variation in gut microbial communities between species than within species. Differences between letters above each barplot denote a significant difference in UniFrac distance (P-value $<0.05)$.

Pairwise UniFrac Distances

Round Goby vs. Yellow Bullhead

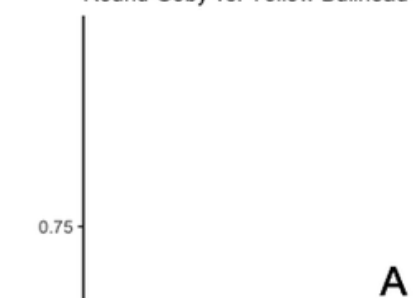

A

B

A

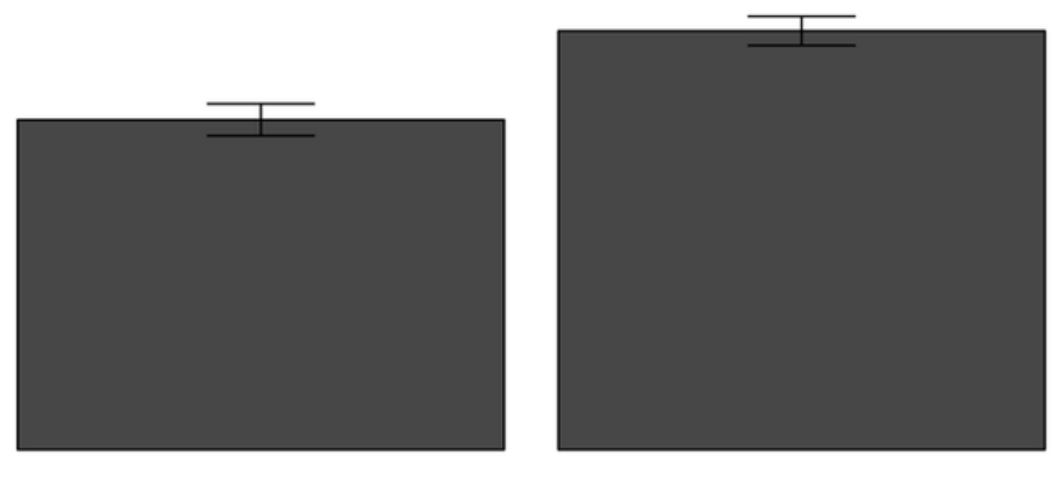

Round Goby vs. Round Goby

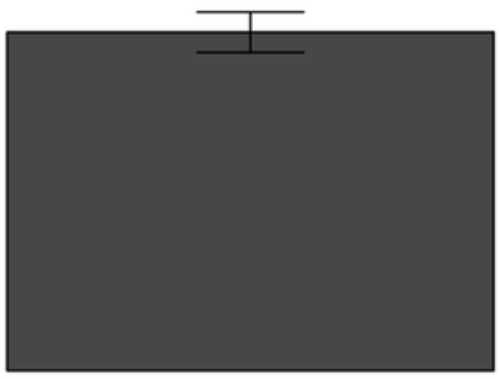




\section{Figure 9}

Pairwise UniFrac distances calculated from the OTU's derived from the Round Goby habitat comparison.

Round Goby gut communities differed between habitats and deepwater (Eagle Wings Island) fish had less within habitat variations than fish in the near shore littoral (Governors Island) Differences between letters above each barplot denote a significant difference in UniFrac distance (P-value $<0.05)$.

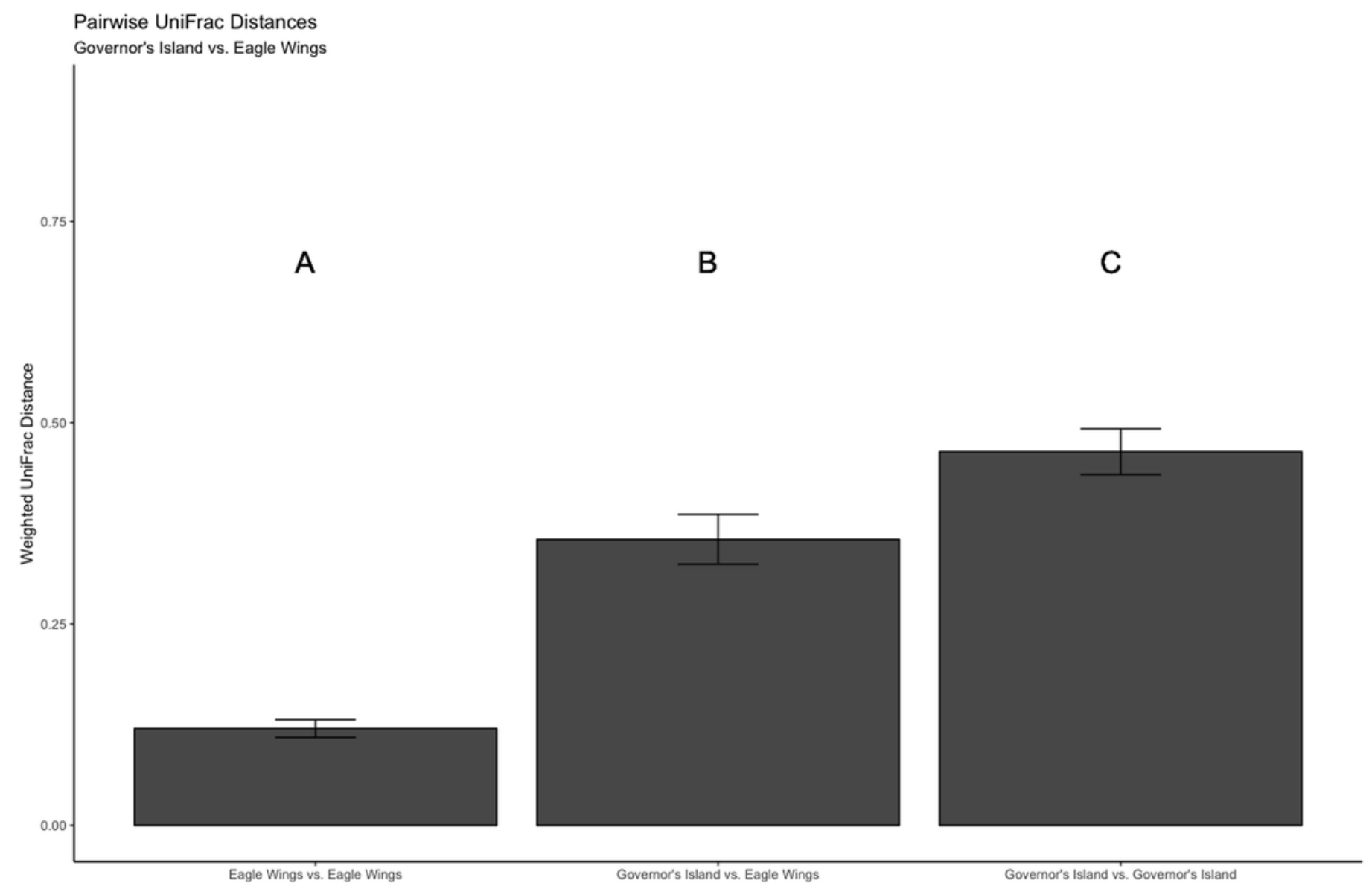




\section{Figure 10}

Relative Gene Abundance of predicted microbial community functions from the species comparison, calculated using Tax4Fun and the Top 20 level one pathway KEGG Orthologs.

There were no significant species differences in microbial community functions pertaining to Environmental Information Processing (A), Cellular Processes (B), Metabolism (C) or Genetic Information Processing (D) for all measured level one KEGG Ortholog pathways (P-values = $0.79,0.86$, and 0.57 , respectively). 


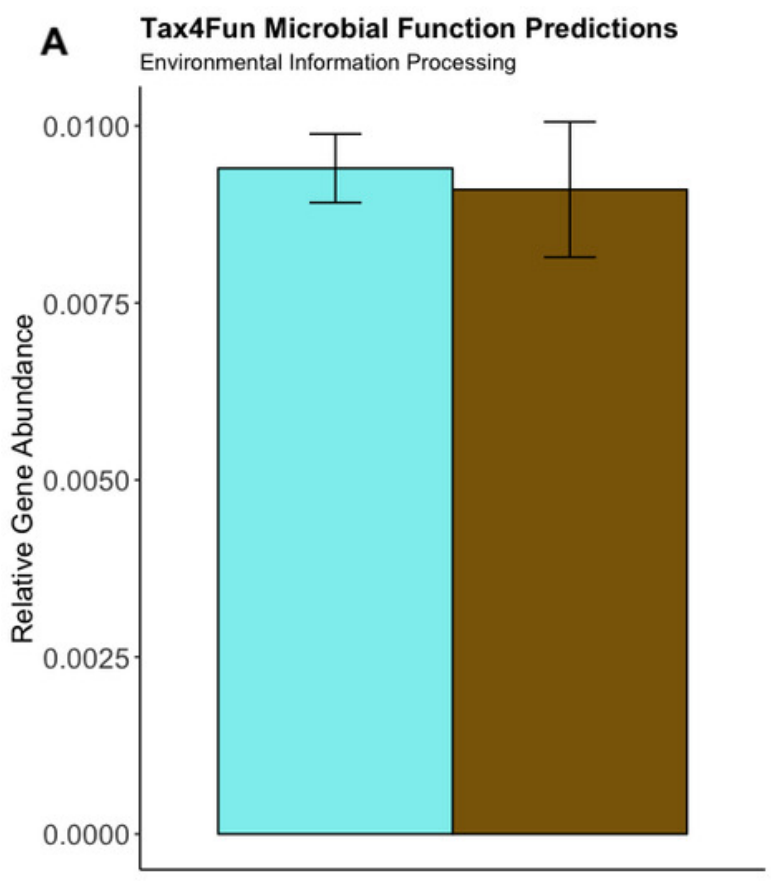

C Tax4Fun Microbial Function Predictions
Metabolism

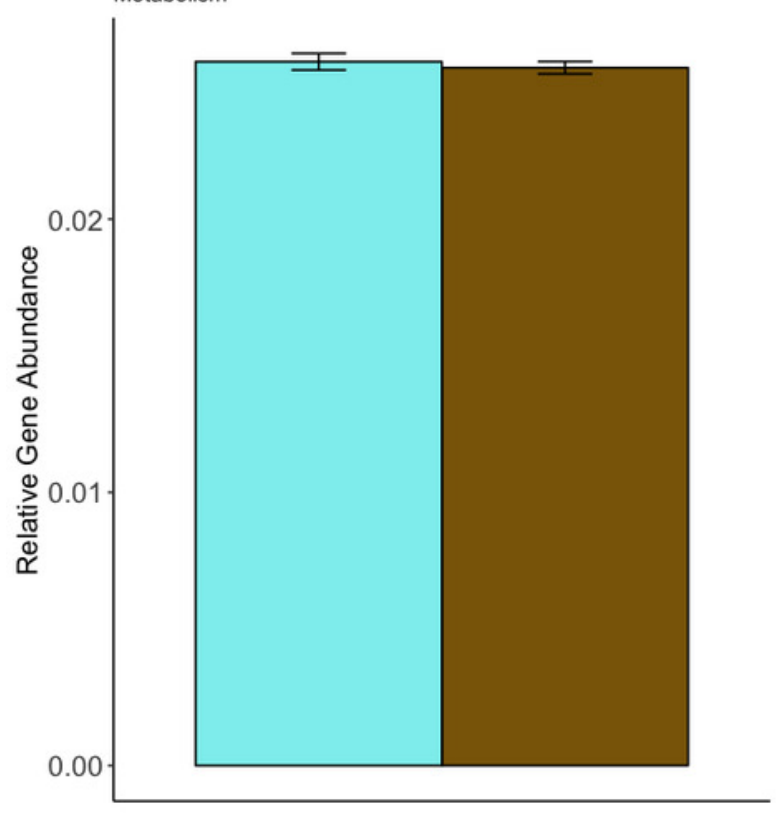

B Tax4Fun Microbial Function Predictions Cellular Processes
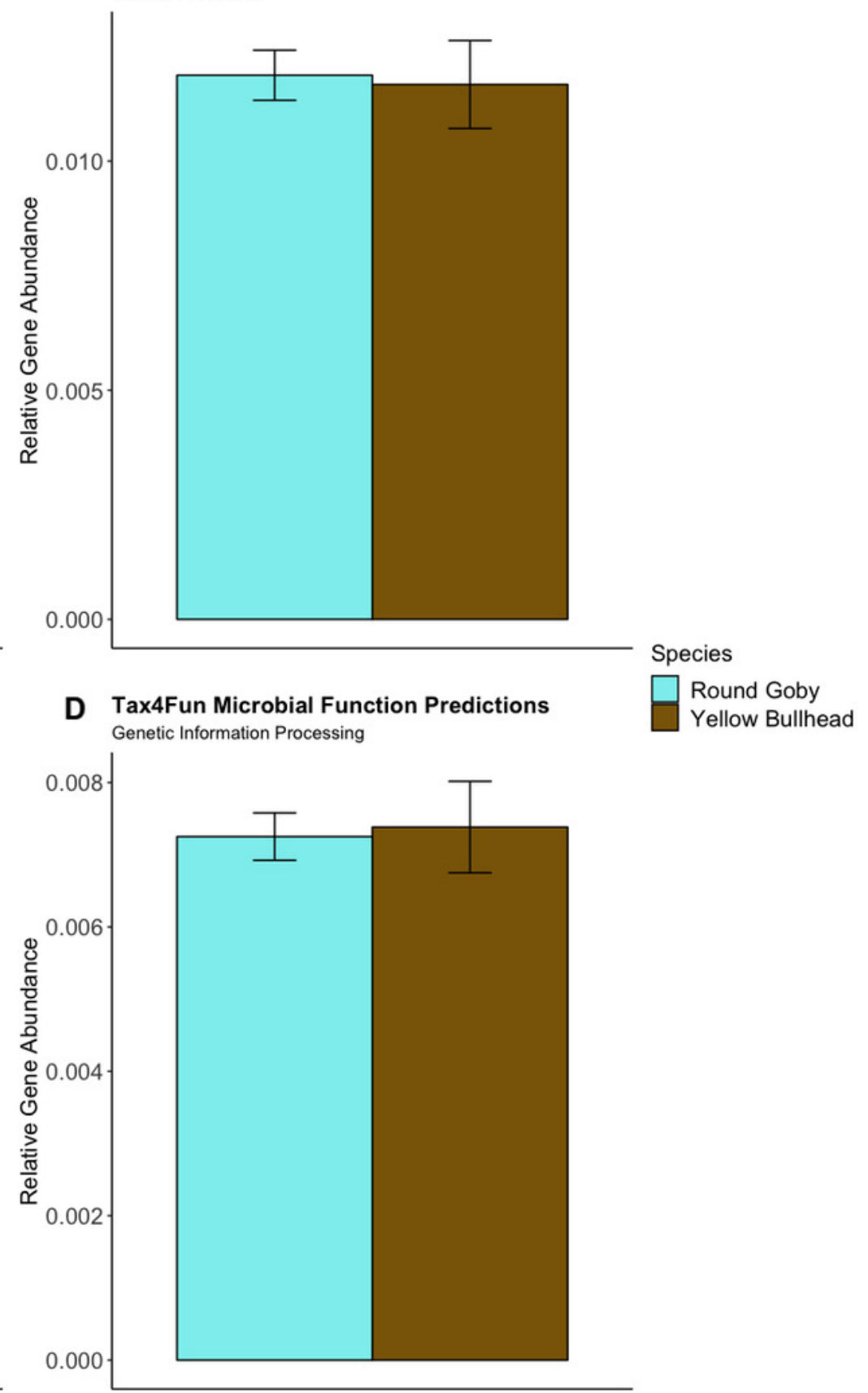


\section{Figure 11}

Relative Gene Abundance of predicted microbial community functions from the habitat comparison, calculated using Tax4Fun and the Top 20 level one pathway KEGG Orthologs.

There were no significant habitat differences in microbial community functions pertaining to Environmental Information Processing (A), Cellular Processes (B), Metabolism (C) or Genetic Information Processing (D) for all measured level one KEGG Ortholog pathways (P-values = $0.11,0.09$, and 0.12 , respectively). 


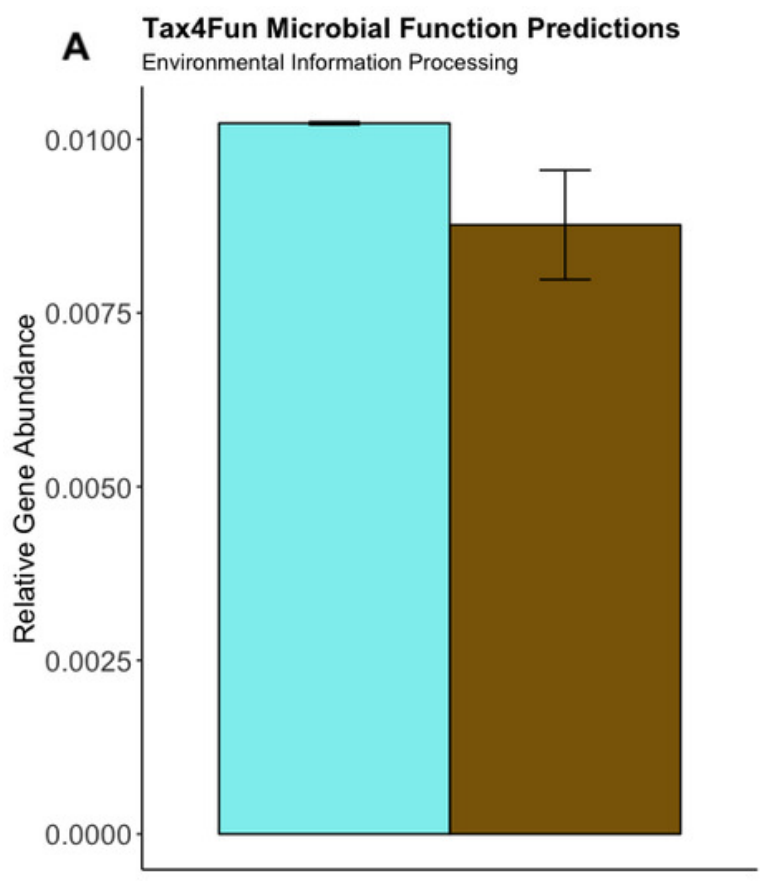

C

Tax4Fun Microbial Function Predictions Metabolism

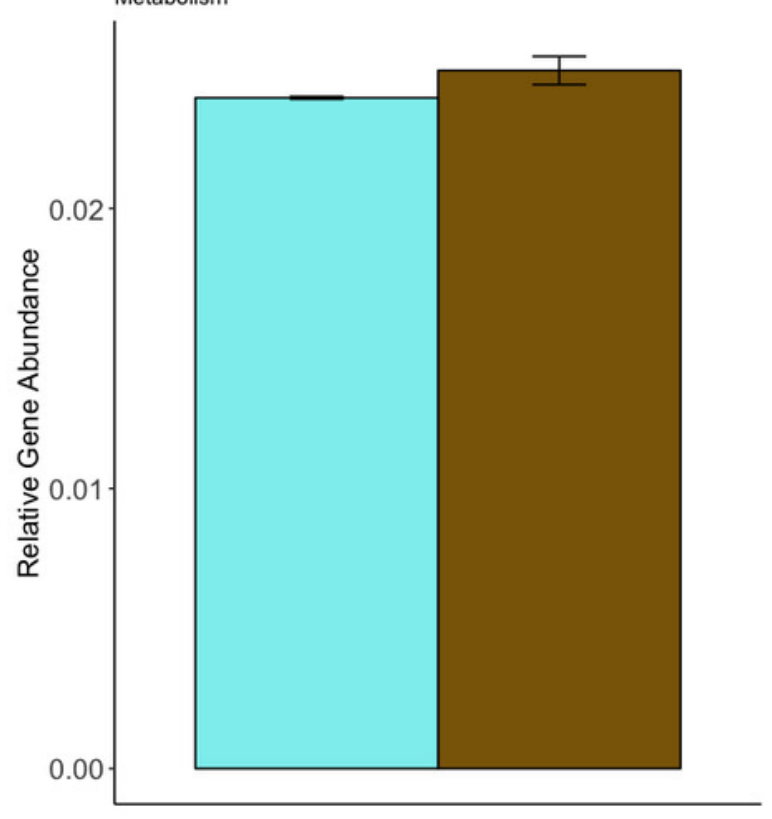

Tax4Fun Microbial Function Predictions Cellular Processes

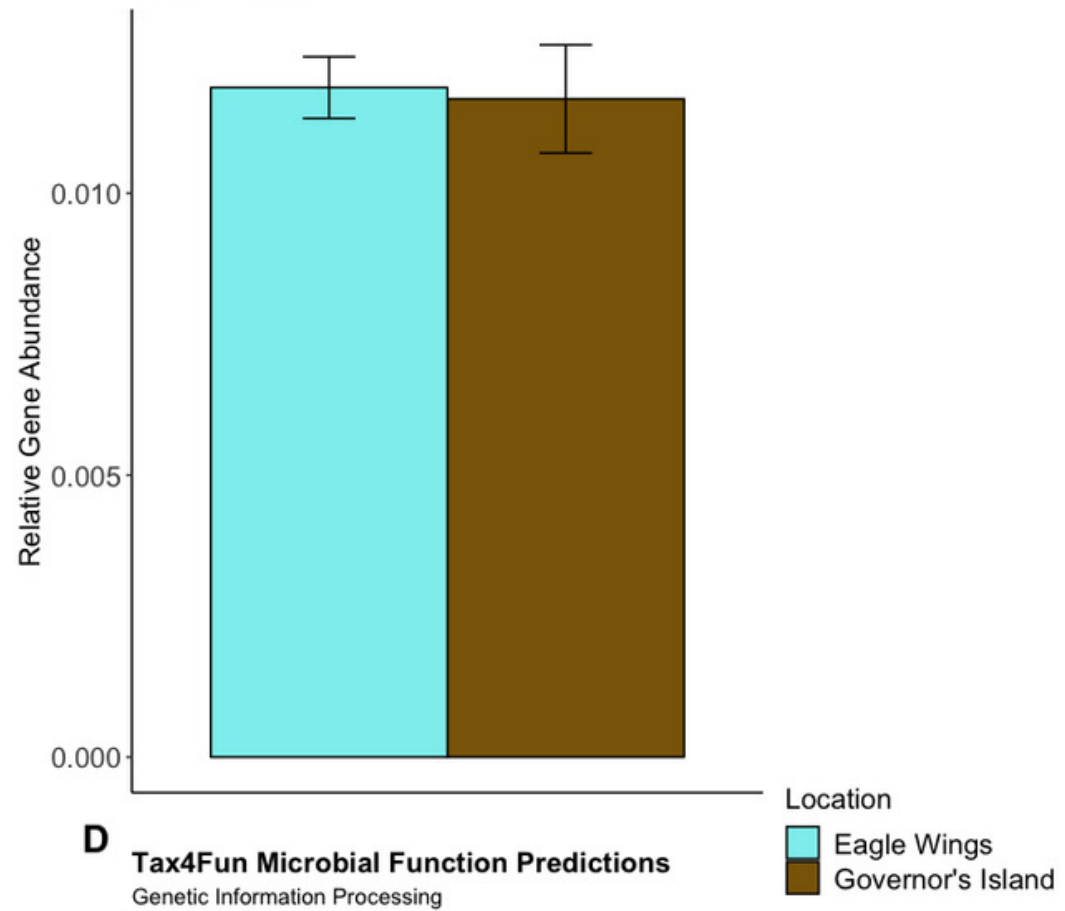

0.000

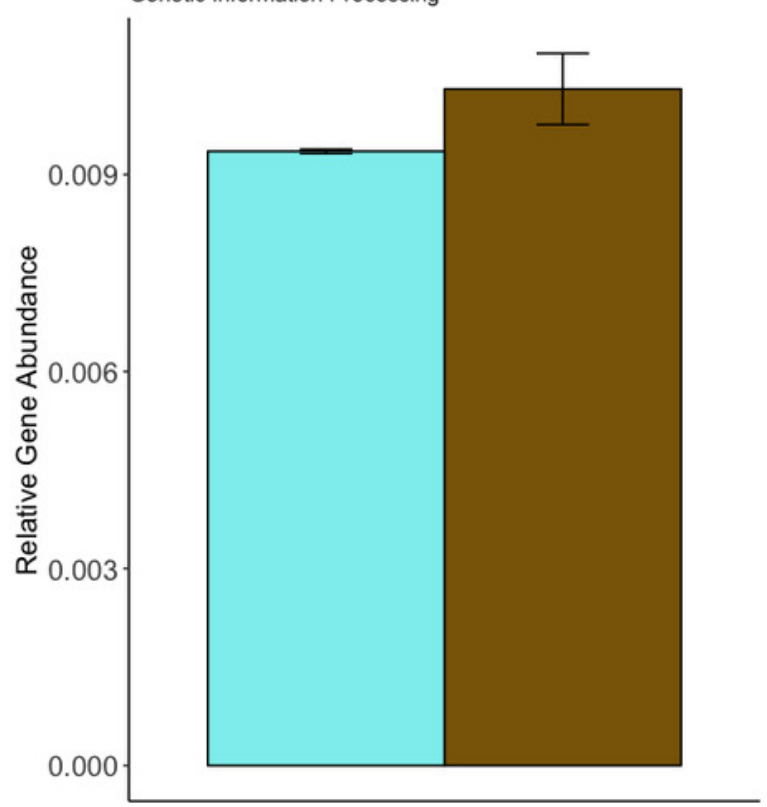




\section{Table $\mathbf{1}$ (on next page)}

Good's Coverage Index calculations for all species (left) and habitat (right) datasets.

Calculations were computed at the normalized singleton sequencing depth $(41,250$ sequences for all samples, 40,972 for RG only samples). All the samples revealed over $99.7 \%$ OTU diversity coverage. $\mathrm{RG}=$ Round Goby, $\mathrm{YBH}=$ Yellow Bullhead, ID = unique sample identification 
1 Table 1: Use of next generation sequencing to compare simple habitat and species level

2 differences in the gut microbiota of an invasive and native freshwater fish species

3

4 Benjamin D. Gallo, Brian F. Leydet Jr., \& John M. Farrell

5 Table 1: Good's Coverage Index calculations for all species (RG vs. YBH; left) and habitat (RG;

6 right) datasets. Calculations were computed at the normalized singleton sequencing depth

7 (41,250 sequences for all samples, 40,972 for RG only samples). All the samples revealed over

$8 \quad 99.7 \%$ OTU diversity coverage. ID = unique sample identification.

9

\begin{tabular}{|c|c|}
\hline \multicolumn{2}{|c|}{ Species Comparison } \\
\hline ID & $\begin{array}{c}\text { Good's } \\
\text { Coverage }\end{array}$ \\
\hline RG025 & 1.000 \\
\hline RG026 & 1.000 \\
\hline RG027 & 1.000 \\
\hline RG029 & 1.000 \\
\hline RG031 & 0.999 \\
\hline RG032 & 1.000 \\
\hline RG033 & 1.000 \\
\hline RG034 & 1.000 \\
\hline RG037 & 1.000 \\
\hline RG038 & 1.000 \\
\hline RG043 & 0.999 \\
\hline RG045 & 1.000 \\
\hline RG048 & 1.000 \\
\hline RG049 & 1.000 \\
\hline RG050 & 1.000 \\
\hline YBH039 & 0.999 \\
\hline YBH041 & 0.999 \\
\hline YBH051 & 0.997 \\
\hline YBH052 & 0.998 \\
\hline YBH053 & 0.999 \\
\hline YBH055 & 0.998 \\
\hline YBH056 & 0.999 \\
\hline
\end{tabular}

11

\begin{tabular}{cc}
\hline \multicolumn{2}{c}{ Habitat Comparison } \\
\hline ID & Good's Coverage \\
RG025 & 1.000 \\
RG026 & 1.000 \\
RG027 & 1.000 \\
RG029 & 1.000 \\
RG031 & 0.999 \\
RG032 & 1.000 \\
RG033 & 1.000 \\
RG034 & 1.000 \\
RG037 & 1.000 \\
RG038 & 1.000 \\
RG043 & 0.999 \\
RG045 & 1.000 \\
RG048 & 1.000 \\
RG049 & 1.000 \\
RG050 & 1.000 \\
\hline
\end{tabular}

10 